\title{
Resummation for the field-theoretical derivation of the negative magnetoresistance
}

\author{
Kenji Fukushima ${ }^{a, b}$ and Yoshimasa Hidaka ${ }^{c, d}$ \\ ${ }^{a}$ Department of Physics, The University of Tokyo, \\ 7-3-1 Hongo, Bunkyo-ku, Tokyo 113-0033, Japan \\ ${ }^{b}$ Institute for Physics of Intelligence, The University of Tokyo, \\ 7-3-1 Hongo, Bunkyo-ku, Tokyo 113-0033, Japan \\ ${ }^{c}$ Theoretical Research Division, Nishina Center, RIKEN, \\ 2-1 Hirosawa, Wako, Saitama 351-0198, Japan \\ ${ }^{d}$ RIKEN iTHEMS, RIKEN, \\ 2-1 Hirosawa, Wako, Saitama 351-0198, Japan \\ E-mail: fuku@nt.phys.s.u-tokyo.ac.jp, hidaka@riken.jp
}

ABSTRACT: We show detailed derivation of the electric conductivity of quark matter at finite temperature and density under a magnetic field. We especially focus on the longitudinal electric conductivity along the magnetic direction and establish the field-theoretical description of the negative magnetoresistance as observed in chiral materials. With increasing magnetic field our microscopic calculation leads to changing behavior from approximately quadratic to asymptotically linear dependence of the electric conductivity, while the magnetic dependence is quadratic in the conventional relaxation time approximation. The presented formulation founds a firm basis for the physical interpretation of the negative magnetoresistance in terms of the particle and the hydrodynamic contributions, as well as it offers general methodology applicable for various transport coefficients.

KeYwords: Resummation, Quark-Gluon Plasma, Perturbative QCD

ARXIV EPRINT: 1906.02683 


\section{Contents}

1 Introduction 1

2 Conventions, definitions, and the final results first 4

3 Warm-ups: the Hall conductivity and the transverse conductivity $\quad 7$

$\begin{array}{lll}3.1 & \text { Real-time propagators } & 7\end{array}$

3.2 Loop integrals 8

4 Longitudinal conductivity 11

$\begin{array}{ll}\text { 4.1 Boltzmann equations and the formal solution } & 12\end{array}$

$\begin{array}{lll}4.2 & \text { Collision terms } & 15\end{array}$

$\begin{array}{lll}4.3 & \text { Matrix elements } & 20\end{array}$

4.4 Numerical results and approximation checks 23

4.5 Asymptotics at strong magnetic field toward the LLLA 26

$\begin{array}{llr}5 & \text { Diagrammatic approach } & \mathbf{2 8}\end{array}$

$\begin{array}{ll}5.1 R / A \text { basis } & 28\end{array}$

5.2 Expansion of the dressed propagator 29

5.3 Resummation of the vertex function and the linearized Boltzmann equations 31

6 Conclusions $\quad 36$

\section{Introduction}

Chiral magnetic effect (CME) was originally proposed as a measurable probe to topologically nontrivial excitations in relativistic nucleus-nucleus collisions [1]. The theoretical framework has been elaborated with recognition of "chiral chemical potential" introduced later in ref. [2]. In the formulation with the chiral chemical potential the theoretical link between the CME and the chiral anomaly in quantum chromodynamics (QCD) has become transparent in a similar fashion to an axial counterpart that had been known [3] and is called the chiral separation effect nowadays. The idea can be translated to simpler setups with the chiral chemical potential replaced by electromagnetic background fields $[4,5]$, which has opened a new opportunity to observe the chiral anomaly not necessarily in QCD but in more accessible experiments [6]. For a fairly complete list of references (especially earlier works), see a recent review [7]. Readers can also consult an essay of ref. [8] for developments of the idea.

One clear signature for the CME is supposed to be the negative magnetoresistance as proposed in ref. [5] and it has been confirmed experimentally in ref. [7] followed by independent experiments with Dirac semimetals $[9,10]$ and Weyl semimetals $[11,12]$. The negative 
magnetoresistance refers to decreasing electric resistance, $\rho$, or equivalently increasing electric conductivity, $\sigma=1 / \rho$, along the magnetic direction with increasing magnetic field $B$. Intuitively, as derived in ref. [7], one can understand the physical mechanism as follows: the chirality production is enhanced as $\propto E B$ by the chiral anomaly through the Schwinger mechanism in the presence of parallel electromagnetic fields [4, 13, 14] (see also ref. [15] for a review), and successively, the chiral magnetic effect adds an extra conductivity term $\propto(E B) B \propto B^{2}$.

Although the above-mentioned mechanism is robust, one may think that $\rho$ or $\sigma$ could be anyway affected by $B$. For instance, a hot-QCD medium can be approximated as a gas of hadron resonances, in which, superficially, the chiral anomaly is irrelevant. Nevertheless, it has been revealed that the electric charge fluctuation in this model is significantly enhanced with increasing $B$ especially at finite chemical potential $\mu$ [16], which also suggests significant enhancement in the electric conductivity. One might be then tempted to make some unfavorable statements about the magnetoresistance and the chiral anomaly. In principle, however, the model could implicitly incorporate the effect of the chiral anomaly, and it is impossibly difficult to draw a solid conclusion from such model studies and crude approximations.

To establish the physical interpretation, therefore, it is desirable to complete fieldtheoretical calculations for $\rho$ or $\sigma$ taking account of full nonlinear dependence on $B$. The starting point for the conductivity calculation should be the Kubo formula, but as we will discuss in great details, some resummation is quite often indispensable and literal calculations might be formidably cumbersome. Then, it would be one of the most tractable approaches to utilize the kinetic equation, as adopted also in ref. [5]. In the estimate previously made in ref. [5], however, the Chiral Kinetic Theory (CKT) [17-34] was assumed, in which the Dirac spin structure reduces to the Berry curvature under the adiabatic approximation. Besides, in ref. [5] and in the theoretical argument in ref. [7], the relaxation-time approximation was used with a constant relaxation time. These treatments are appropriate for small $B$, but are not necessarily so for large $B$.

If $B$ (multiplied by a typical electric charge) is the largest energy scale in systems with charged particles, the lowest Landau level (LLL) would make a dominant contribution to physical observables. The theoretical calculation is then feasible enough in the approximation to take the LLL only (i.e., LLLA). Moreover, we point out that the whole theoretical description would be far cleaner in QCD matter rather than in condensed matter systems; for the former the fundamental theory is $\mathrm{QCD}$, but for the the latter quantum electrodynamics (QED) cannot be directly employed. In fact, various transport coefficients in QCD matter have been estimated in the LLLA of QCD that is justified with assumed hierarchy, $\sqrt{q B} \gg T \gg g T$, where $q$ is an electric charge and $g$ is a QCD charge of quarks. The heavy-quark diffusion constant is found in ref. [35], the electric conductivity in refs. [36, 37], and the viscosity in ref. [38], etc.

Usually the LLLA is a good approximation as long as the magnetic scale is large enough. For the longitudinal electric conductivity, however, the LLLA is broken down due to phase space and it is insufficient for the negative magnetoresistance at intermediate strength of the magnetic field. As pointed out in ref. [35], strong- $B$ fields reduce quark 
dispersions from $(3+1)$ - to $(1+1)$-dimensional dynamics, and quarks thus cannot scatter if they are massless; otherwise, the energy-momentum conservations would be violated. This implies that the electric conductivity in the LLLA diverges as $m_{f}$, a mass of $f$ flavor quark, goes to zero, i.e., $\sigma_{\text {LLLA }}\left(m_{f} \rightarrow 0\right) \rightarrow \infty$. We will circumvent diverging $\sigma$ by considering higher Landau levels. Specifically, in the present work, we assume a different regime of relevant scales, i.e., $T \gtrsim \sqrt{q B} \gg g T$, which is more relevant for realistic magnetic situations. In this regime the magnetic field is no longer the largest scale, but still we require the last inequality in order to neglect thermal excitation effects as compared to the magnetically induced gap. Here let us make a comment on a subtle point about the massless limit. As we will closely discuss later, in the strict massless limit, the axial charge increases linearly with time, and the physical conductivity diverges even beyond the LLLA. Nevertheless, the transport coefficient used for the hydrodynamics (in which the conserved axial charge makes a hydrodynamic mode) is still finite, that is what we are computing in this work. The point is the time scale; if we take the long time (i.e., small frequency) limit first and approach the massless limit, the conductivity would diverge. If we first adopt small masses and then take the hydrodynamic limit, the conductivity would remain finite as we will demonstrate. Such a seeming difference appears from two hydrodynamic regimes, and the hydrodynamical evolution in respective frameworks should lead to the same renormalized conductivity.

We would note one more advantage of performing the calculation in QCD on top of its being a theoretically clean setup. There are some numerical results available from firstprinciples simulations by means of lattice discretized formulation of QCD, namely, the lattice-QCD simulations. The latest quenched lattice-QCD results are $0.2 C_{\mathrm{em}} \lesssim \sigma / T \lesssim$ $0.7 C_{\mathrm{em}}$ for $T=1.1 T_{\mathrm{c}}$ where $C_{\mathrm{em}}:=\sum_{f} q_{f}^{2}$ and $T_{\mathrm{c}}$ is the QCD critical temperature [39]. See also refs. [40-42] for earlier results. These estimated values are from the simulations at $T \neq$ 0 and $B=0$, but the order of magnitude is expected to be the same as our calculations for $T \gtrsim \sqrt{q B} \gg g T$ if the magnetic dependence of $\sigma$ is smooth enough. The consistency would support validity of our calculations and we will numerically confirm this expectation later.

Besides physics interest, as we will discuss in this work, the theoretical framework of the resummation scheme is very interesting on its own. There are two major technical advances reported in this paper. One is a clear prescription for the hydrodynamic zero modes that appear at finite density associated with conserved quantities. The other is a field-theoretical derivation of the Boltzmann equations as a result of the resummation on the vertex. Since the latter particularly needs judicious transformations of expressions, we first discuss physics parts based on the assumed Boltzmann equations, and then proceed into their step-by-step derivations.

This paper is organized as follows. In section 2 we summarize our conventions and field-theoretical definitions of physical quantities of our interest. As warm-up exercises in section 3 we perform explicit calculations for the Hall conductivity and give discussions on the transverse conductivity which is vanishing at the one-loop order. Section 4 is the central part of this paper consisting of subsections devoted to the methods, the numerical results, and the physics discussions. To fill in the gap between the Kubo formula and the Boltzmann equations, we explicate step-by-step derivations in section 5 . We finally make conclusions in section 6 . 


\section{Conventions, definitions, and the final results first}

For readers to have better accessibility to the contents, we will show our final results first here. Before doing this, let us summarize the conventions and the definitions used in this work. Bearing them in mind, readers can understand which physical quantity we are calculating and what the final results are, before the subsequent technical discussions. We will gradually proceed to technical details in later sections, first the one-loop integral in section 3, next the resummed kinetic description in section 4, and finally the full diagrammatic derivation in section 5 .

Our convention of the Lorentz four vector assumes the Minkowski metric, $\eta_{\mu \nu}=$ $\operatorname{diag}(1,-1,-1,-1)$. We choose the covariant derivative as $D_{\mu}=\partial_{\mu}+\mathrm{i} q_{f} A_{\mu}$ where $q_{f}$ is the electric charge carried by quark flavor $f$ and $A_{\mu}$ is the electromagnetic gauge field. In this paper, we treat the gluonic degrees of freedom as well, but the background field is only electromagnetic. We often use a sloppy but intuitive notation for the contravariant components of the four momentum vector as $p^{\mu}=\left(p^{0}, p^{1}, p^{2}, p^{3}\right)=\left(\varepsilon, p_{x}, p_{y}, p_{z}\right)$ using the coordinates $x, y$, and $z$, and sometimes switch the latter back to the former for notational convenience.

The physical observable of our utmost interest in this work is the electric conductivity, which is given by the Kubo formula as follows:

$$
\sigma^{i j}=\lim _{k_{0} \rightarrow 0} \lim _{\boldsymbol{k} \rightarrow \mathbf{0}} \frac{1}{2 \mathrm{i} k_{0}}\left[\Pi_{R}^{i j}(k)-\Pi_{A}^{i j}(k)\right]
$$

with $i, j$ taking spatial coordinates, $x, y, z$. Here, the order of two limits is important; $\boldsymbol{k} \rightarrow \mathbf{0}$ is taken first and $k_{0} \rightarrow 0$ next. In the above expression $\Pi_{R}^{i j}(k)$ and $\Pi_{A}^{i j}(k)$ are the retarded and advanced polarization functions, respectively, defined by

$$
\begin{aligned}
& \Pi_{R}^{i j}(k):=\mathrm{i} \int \mathrm{d}^{4} x \mathrm{e}^{\mathrm{i} k \cdot x} \theta(t)\left\langle\left[j^{i}(x), j^{j}(0)\right]\right\rangle, \\
& \Pi_{A}^{i j}(k):=-\mathrm{i} \int \mathrm{d}^{4} x \mathrm{e}^{\mathrm{i} k \cdot x} \theta(-t)\left\langle\left[j^{i}(x), j^{j}(0)\right]\right\rangle .
\end{aligned}
$$

It is crucially important to note that $j^{i}$ is not exactly the electric current if a finite density is coupled. This is so because, as we will see later, zero modes or hydrodynamic modes make the inversion operation ill-defined and we should subtract them (which should be included in dynamics of hydrodynamic modes with the calculated conductivity, not in the conductivity itself). Specifically, the above $j^{i}$ should be given by

$$
j^{i}=j_{\mathrm{em}}^{i}-\frac{n_{e} T^{0 i}}{\mathcal{E}+\mathcal{P}_{i}},
$$

where $T^{\mu \nu}$ represents the energy momentum tensor, $n_{e}$ the net electric charge density, $\mathcal{E}:=\left\langle T^{00}\right\rangle$ the energy density, and $\mathcal{P}_{i}:=\left\langle T^{i i}\right\rangle$ the pressure. Because $j^{i}$ is an operator, the numerator of the correction term is an operator $\propto T^{0 i}$, while the denominator is an expectation value, namely, the enthalpy of the system. In QCD with multiple quark flavors the electric current is a sum of contributions from all flavors $f$, i.e.,

$$
j_{\mathrm{em}}^{i}=\sum_{f} q_{f} \bar{\psi}_{f} \gamma^{i} \psi_{f}
$$

If the net electric charge is zero (as is the case at zero density), $j^{i}=j_{\mathrm{em}}^{i}$ simply holds. 
Now, let us explain the decomposition of the electric conductivity tensor into the longitudinal component and others. The external magnetic field introduces a preferred direction, and we decompose the anisotropic tensor structure using $\hat{B}^{i}:=B^{i} /|\boldsymbol{B}|$ as

$$
\sigma^{i j}=\sigma_{H} \epsilon^{i j k} \hat{B}^{k}+\sigma_{\|} \hat{B}^{i} \hat{B}^{j}+\sigma_{\perp}\left(\delta^{i j}-\hat{B}^{i} \hat{B}^{j}\right),
$$

where $\sigma_{H}, \sigma_{\|}$, and $\sigma_{\perp}$ represent the Hall conductivity, the longitudinal conductivity, and the transverse conductivity, respectively. Without loss of generality we can identify the magnetic field direction with the $z$ axis, so that we explicitly identify these conductivities as $\sigma_{H}=\sigma^{12}=-\sigma^{21}, \sigma_{\|}=\sigma^{33}$, and $\sigma_{\perp}=\sigma^{11}=\sigma^{22}$.

So far, all the formulas are general, and we do not impose any physical condition yet. In this paper, as we declared in the introduction, we work only in the weak coupling case and mostly consider a regime in which the following hierarchy is satisfied:

$$
T \gtrsim \sqrt{\left|q_{f} B\right|} \gg g T,
$$

where $f$ is an arbitrary flavor, $g$ is the quark-gluon coupling constant (which is assumed to be small in the weak coupling case), and $T$ is the temperature. We note that the above hierarchy is not a sufficient condition for the LLLA because of the phase space suppression for light fermions, as was also emphasized in the introduction. It is, therefore, important to take account of full Landau levels as we will do in this work. One may then wonder why such a lower bound in eq. (2.7) is needed though we consider all the Landau levels. This is because, as we will explicitly see later, we make an approximation not to include the finite- $T$ induced self-energy $\sim g^{2} T^{2}$ in the quark propagator, which is justified if the $B$ induced gap is much larger, i.e., $\left|q_{f} B\right| \gg g^{2} T^{2}$ that is nothing but the second inequality in eq. (2.7). A side remark is that, as argued in ref. [35], the lowest Landau level is not gapped with $\left|q_{f} B\right|$, but still, no finite- $T$ term appears at all in $(1+1)$ dimensions, so that our approximated quark propagator can be valid. In contrast, the upper bound by $T$ has a looser origin. Later, we will limit our calculation to the lowest order perturbation in $g$ (i.e., $1 \leftrightarrow 2$ processes), but if $B$ becomes too larger than $T$, higher order processes might not be dropped. To stay in the safe side we impose this upper bound. Once the scattering processes are fixed, however, the calculation itself does not break down even for arbitrarily large $B$, and this large- $B$ analysis is what we will perform in our discussions on the LLLA later.

We make a short remark about the sphaleron transition which may in principle contribute to the electric conductivity. In QCD at weak coupling, however, the sphaleron transition rate is as small as $\alpha_{s}^{5}$, and we can safely neglect it. Thus, in this work, we focus on the perturbative contribution only.

Now that we articulated what we want to calculate under which condition, then, we shall present our final results in advance. The easiest to obtain is the Hall conductivity, that is,

$$
\sigma_{H}=\frac{n_{e}}{B},
$$

as will be elaborated in the next warm-up section, where we will deal with explicit expressions for the propagators in the magnetic field. The transverse conductivity is vanishing at 


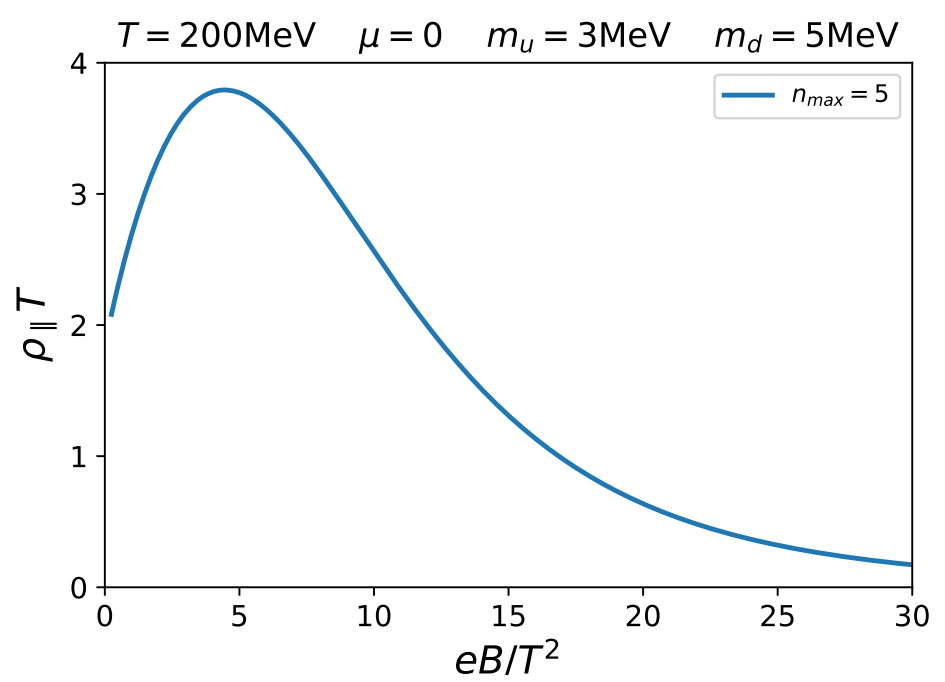

Figure 1. Negative magnetoresistance; $\rho_{\|}$decreases with increasing magnetic field $e B$. The temperature is $T=200 \mathrm{MeV}$ and the density is zero, and $n_{\max }=5$ represents the highest Landau level taken into the calculation.

the one-loop level and, as discussed in the next section, higher-loop terms are suppressed by the magnetic field parametrically as

$$
\frac{\sigma_{\perp}}{T} \sim \frac{g^{2} T^{2}}{\left|q_{f} B\right|}
$$

Since the transverse conductivity is small in our hierarchy regime (2.7), we would not try to quantify $\sigma_{\perp}$ any further.

The most interesting is the longitudinal electric conductivity $\sigma_{\|}$or the resistance $\rho_{\|}$, which is made dimensionless with the temperature as

$$
\rho_{\|} T:=\frac{1}{\left(\sigma_{\|} / T\right)}
$$

The negative magnetoresistance signifies decreasing behavior of $\rho_{\|}$with increasing magnetic field, and this is precisely what we finally find in our calculation as shown in figure 1 . We note that $e$ of $e B$ on the horizontal axis is the (positive) elementary charge, and in this calculation, $u$ and $d$ quark flavors are included with $q_{u}=\frac{2}{3} e$ and $q_{d}=-\frac{1}{3} e$. As is clear from the plot, we have confirmed the negative magnetoresistance from field-theoretical calculations without assuming anything special about the chirality production and the topological transport. Here, we should emphasize that no assumption does not mean no chiral anomaly. Actually, if one solves the Dirac equation in the presence of electromagnetic background, the chiral anomaly should be automatically incorporated. In gauge theories fermions are bilinear, so that fermion integrals can be one-loop exact apart from gauge fluctuations. 


\section{Warm-ups: the Hall conductivity and the transverse conductivity}

For diagrammatic calculations the elementary building blocks are the propagator and the vertices. In the presence of the magnetic field even the free propagator takes a cumbersome form, as we cope with in this section. We will discuss the resummed vertices in the next section. Here, we will perform a one-loop calculation using explicit expressions of the propagators as warm-up exercises, and we will find the Hall conductivity as well as the suppressed transverse conductivity.

\subsection{Real-time propagators}

We here adopt the real-time Schwinger-Keldysh formalism in the Keldysh basis. The propagators in the Keldysh basis and the standard ones on the Schwinger-Keldysh paths $(1,2)$ are transformed through the following relations:

$$
\begin{aligned}
& S_{R R}^{f}:=\frac{1}{4}\left\langle\left(\psi_{f 1}+\psi_{f 2}\right)\left(\bar{\psi}_{f 1}+\bar{\psi}_{f 2}\right)\right\rangle=\frac{1}{4}\left(S_{11}^{f}+S_{12}^{f}+S_{21}^{f}+S_{22}^{f}\right)=-\mathrm{i} S_{K}^{f}, \\
& S_{R A}^{f}:=\frac{1}{2}\left\langle\left(\psi_{f 1}+\psi_{f 2}\right)\left(\bar{\psi}_{f 1}-\bar{\psi}_{f 2}\right)\right\rangle=\frac{1}{2}\left(S_{11}^{f}-S_{12}^{f}+S_{21}^{f}-S_{22}^{f}\right)=-\mathrm{i} S_{R}^{f}, \\
& S_{A R}^{f}:=\frac{1}{2}\left\langle\left(\psi_{f 1}-\psi_{f 2}\right)\left(\bar{\psi}_{f 1}+\bar{\psi}_{f 2}\right)\right\rangle=\frac{1}{2}\left(S_{11}^{f}+S_{12}^{f}-S_{21}^{f}-S_{22}^{f}\right)=-\mathrm{i} S_{A}^{f}, \\
& S_{A A}^{f}:=\left\langle\left(\psi_{f 1}-\psi_{f 2}\right)\left(\bar{\psi}_{f 1}-\bar{\psi}_{f 2}\right)\right\rangle=\left(S_{11}^{f}-S_{12}^{f}-S_{21}^{f}+S_{22}^{f}\right)=0 .
\end{aligned}
$$

The last identify generally holds, which is understood from the spectral representation of the propagators. Now, to proceed further, we need expressions for the free propagators under the magnetic field $B$. The retarded/advanced propagator is given by a sum over the Landau levels labeled by $n$ as

$$
S_{R / A}^{f}(p)=\sum_{n=0}^{\infty} \frac{-S_{n}^{f}(p)}{p_{0}^{2}-\varepsilon_{f n}^{2} \pm \mathrm{i} \epsilon p_{0}}=\sum_{n=0}^{\infty} \frac{-S_{n}^{f}(p)}{p_{\|}^{2}-m_{f n}^{2} \pm \mathrm{i} \epsilon p_{0}},
$$

where $R$ corresponds to + and $A$ to - in front of $\mathrm{i} \epsilon p_{0}$. The Landau quantized energy dispersion is $\varepsilon_{f n}=\sqrt{p_{z}^{2}+2\left|q_{f} B\right| n+m_{f}^{2}}$. To go to the last expression above, we defined $m_{f n}^{2}:=2\left|q_{f} B\right| n+m_{f}^{2}$ and the transverse and the longitudinal momenta, $p_{\perp}^{\mu}=\left(0, p_{x}, p_{y}, 0\right)$ and $p_{\|}^{\mu}=\left(p_{0}, 0,0, p_{z}\right)$, in accord to the convention with $\boldsymbol{B}$ chosen along the $z$ axis.

The numerator $S_{n}^{f}(p)$ has Dirac index structures decomposed as

$$
S_{n}^{f}(p)=\left(\not p_{\|}+m_{f}\right)\left[P_{+}^{f} A_{n+}\left(4 \xi_{p}^{f}\right)+P_{-}^{f} A_{n-}\left(4 \xi_{p}^{f}\right)\right]+\not p_{\perp} B_{n}\left(4 \xi_{p}^{f}\right)
$$

with $\xi_{p}^{f}:=\left|\boldsymbol{p}_{\perp}\right|^{2} /\left(2\left|q_{f} B\right|\right)$ and

$$
\begin{aligned}
A_{n+}(\xi) & :=2 \mathrm{e}^{-\xi / 2}(-1)^{n} L_{n}(\xi), \\
A_{n-}(\xi) & :=2 \mathrm{e}^{-\xi / 2}(-1)^{n-1} L_{n-1}(\xi), \\
B_{n}(\xi) & :=4 \mathrm{e}^{-\xi / 2}(-1)^{n-1} L_{n-1}^{(1)}(\xi) .
\end{aligned}
$$

Here, $L_{n}(\xi)=L_{n}^{(0)}(\xi)$, and $L_{n}^{(\alpha)}(\xi)$ represents the generalized Laguerre Polynomials [43]. The above form is the same one as used in ref. [44]. For the propagator and related integrals, 
see also refs. $[45,46]$. In the above expression $P_{ \pm}^{f}$ represents the projection operator defined by $P_{ \pm}^{f}:=\frac{1}{2}\left[1 \pm \operatorname{sgn}\left(q_{f} B\right) \mathrm{i} \gamma^{1} \gamma^{2}\right]$. For convenience to treat the projection operators, let us introduce,

$$
\gamma^{ \pm}:=\frac{1}{2}\left(\gamma^{1} \pm \mathrm{i} \gamma^{2}\right)
$$

which satisfy $\left(\gamma^{ \pm}\right)^{2}=0$ and $\gamma^{ \pm} \gamma^{\mp}=-\left(1 \pm \mathrm{i} \gamma^{1} \gamma^{2}\right) / 2$, with which the projection operators can be represented as $P_{ \pm \operatorname{sgn}\left(q_{f} B\right)}^{f}=-\gamma^{ \pm} \gamma^{\mp}$ and we see $\gamma^{ \pm} P_{\mp \operatorname{sgn}\left(q_{f} B\right)}^{f}=\gamma^{ \pm}$follows. In our later calculations we will make use of these relations.

We note that the propagators must satisfy the fluctuation dissipation relation, which is expressed as

$$
S_{K}^{f}(p)=\left[\frac{1}{2}-n_{F}\left(p_{0}-\mu_{f}\right)\right]\left[S_{R}^{f}(p)-S_{A}^{f}(p)\right]=\mathrm{i}\left[\frac{1}{2}-n_{F}\left(p_{0}-\mu_{f}\right)\right] \rho_{f}(p) .
$$

Here, $n_{F}\left(p_{0}-\mu_{f}\right)$ is the Fermi-Dirac distribution function with an argument shifted by a chemical potential $\mu_{f}$ for $f$-flavor quark. The spectral function is defined by $\mathrm{i} \rho_{f}(p):=$ $S_{R}^{f}(p)-S_{A}^{f}(p)$, which is, for the free propagator,

$$
\rho_{f}(p)=\sum_{n=0}^{\infty} S_{n}^{f}(p)(2 \pi) \operatorname{sgn}\left(p_{0}\right) \delta\left(p_{0}^{2}-\varepsilon_{f n}^{2}\right),
$$

as easily derived from eq. (3.5).

\section{$3.2 \quad$ Loop integrals}

We shall start with the simplest loop integral, that is, the electric charge density, $n_{e}:=\left\langle j^{0}\right\rangle$, for a non-interacting Fermi gas exposed to the magnetic field. In terms of the propagator we write it as

$$
\begin{aligned}
n_{e} & =-\sum_{f} q_{f} \int \frac{\mathrm{d}^{4} p}{(2 \pi)^{4}} \operatorname{tr}\left[\gamma^{0} S_{R R}^{f}(p)\right] \\
& =-N_{\mathrm{c}} \sum_{f} q_{f} \sum_{n=0}^{\infty} \int \frac{\mathrm{d}^{2} p_{\|}}{(2 \pi)^{2}}\left[\frac{1}{2}-n_{F}\left(p_{0}-\mu_{f}\right)\right](2 \pi) \operatorname{sgn}\left(p_{0}\right) \delta\left(p_{0}^{2}-\varepsilon_{f n}^{2}\right) \int \frac{\mathrm{d}^{2} p_{\perp}}{(2 \pi)^{2}} \operatorname{tr}\left[\gamma^{0} S_{n}^{f}(p)\right],
\end{aligned}
$$

which follows from eqs. (3.1), (3.11), and (3.12). The overall minus sign comes from the fermion loop. We can easily take the Dirac trace to find,

$$
\operatorname{tr}\left[\gamma^{0} S_{n}^{f}(p)\right]=4 e^{-4 \xi_{p}^{f} / 2}(-1)^{n} p_{0}\left[L_{n}\left(4 \xi_{p}^{f}\right)-L_{n-1}\left(4 \xi_{p}^{f}\right)\right] .
$$

The transverse integral of the Laguerre Polynomial counts the Landau degeneracy factor as seen from

$$
\int \frac{\mathrm{d}^{2} p_{\perp}}{(2 \pi)^{2}} \mathrm{e}^{-4 \xi_{p}^{f} / 2}(-1)^{n} L_{n}\left(4 \xi_{p}^{f}\right)=\frac{\left|q_{f} B\right|}{2 \pi} \int_{0}^{\infty} d \xi_{p}^{f} \mathrm{e}^{-4 \xi_{p}^{f} / 2}(-1)^{n} L_{n}\left(4 \xi_{p}^{f}\right)=\frac{\left|q_{f} B\right|}{4 \pi}
$$


for $n \geq 0$. Using the above integral expression, we can simplify the electric charge density into the following form:

$$
\begin{aligned}
n_{e} & =-N_{\mathrm{c}} \sum_{f} \sum_{n=0}^{\infty} \alpha_{n} \frac{q_{f}\left|q_{f} B\right|}{\pi} \int \frac{\mathrm{d}^{2} p_{\|}}{(2 \pi)^{2}} p_{0}\left[\frac{1}{2}-n_{F}\left(p_{0}-\mu_{f}\right)\right](2 \pi) \operatorname{sgn}\left(p_{0}\right) \delta\left(p_{0}^{2}-\varepsilon_{f n}^{2}\right) \\
& =N_{\mathrm{c}} \sum_{f} \sum_{n=0}^{\infty} \alpha_{n} \frac{q_{f}\left|q_{f} B\right|}{2 \pi} \int \frac{\mathrm{d} p_{z}}{2 \pi}\left[n_{F}\left(\varepsilon_{f n}-\mu_{f}\right)-n_{F}\left(\varepsilon_{f n}+\mu_{f}\right)\right] .
\end{aligned}
$$

This is exactly the expression obtained by standard procedures to replace the phase space integration with the Landau level sum. Here, as usual, we introduced the spin degeneracy factor $\alpha_{n}$ defined by

$$
\alpha_{n}= \begin{cases}1 & (n=0) \\ 2 & (n>0)\end{cases}
$$

appearing from two Laguerre polynomials in eq. (3.14). This factor takes care of the fact that the LLL at $n=0$ has only one spin state.

We are now ready for going into the next exercise of loop integrals. In the Keldysh basis, we can write the conductivity at the one loop level or the polarization tensor as

$$
\begin{aligned}
\Pi_{R}^{\mu \nu}(k)= & \sum_{f}(-1) \mathrm{i} q_{f}^{2} \int \frac{\mathrm{d}^{4} p}{(2 \pi)^{4}} \operatorname{tr}\left[\gamma^{\mu} S_{R R}^{f}(k+p) \gamma^{\nu} S_{A R}^{f}(p)\right] \\
& +\sum_{f}(-1) \mathrm{i} q_{f}^{2} \int \frac{\mathrm{d}^{4} p}{(2 \pi)^{4}} \operatorname{tr}\left[\gamma^{\mu} S_{R A}^{f}(k+p) \gamma^{\nu} S_{R R}^{f}(p)\right] .
\end{aligned}
$$

For transport coefficients we need to evaluate the above polarization tensor in the $\boldsymbol{k} \rightarrow \mathbf{0}$ limit. For this, we first take the $\boldsymbol{k}_{\perp} \rightarrow \mathbf{0}$ limit, under which the trace with the propagator numerators leads to

$$
\begin{aligned}
\lim _{\perp \rightarrow 0} \operatorname{tr}\left[\gamma^{-} S_{n}^{f}(p+k) \gamma^{+} S_{m}^{f}(p)\right] \\
=4 \mathrm{e}^{-4 \xi_{p}^{f}}(-1)^{n-m} \operatorname{tr}\left\{\gamma^{-}\left[\left(\not p_{\|}+\not k_{\|}+m_{f}\right)\left(P_{+}^{f} L_{n}\left(4 \xi_{p}^{f}\right)-P_{-}^{f} L_{n-1}\left(4 \xi_{p}^{f}\right)\right)-2 \not p_{\perp} L_{n-1}^{(1)}\left(4 \xi_{p}^{f}\right)\right]\right. \\
\left.\quad \times \gamma^{+}\left[\left(\not_{\|}+m_{f}\right)\left(P_{+}^{f} L_{m}\left(4 \xi_{p}^{f}\right)-P_{-}^{f} L_{m-1}\left(4 \xi_{p}^{f}\right)\right)-2 \not p_{\perp} L_{m-1}^{(1)}\left(4 \xi_{p}^{f}\right)\right]\right\} \\
=8 \mathrm{e}^{-4 \xi_{p}^{f}}(-1)^{n-m+1}\left[\left(p_{\|}+k_{\|}\right) \cdot p_{\|}-m_{f}^{2}\right] \\
\quad \times\left[\theta\left(q_{f} B\right) L_{n}\left(4 \xi_{p}^{f}\right) L_{m-1}\left(4 \xi_{p}^{f}\right)+\theta\left(-q_{f} B\right) L_{n-1}\left(4 \xi_{p}^{f}\right) L_{m}\left(4 \xi_{p}^{f}\right)\right]
\end{aligned}
$$

where we used $\gamma^{ \pm}$of eq. (3.10) instead of the coordinate basis. We note that we used $\operatorname{tr}\left[\gamma^{-} p_{\perp} \gamma^{+} p_{\perp}\right]=0$ to arrive at the last expression. Then, we can immediately take the Dirac trace and perform the transverse momentum integration to get

$$
\begin{aligned}
& \lim _{\boldsymbol{k}_{\perp} \rightarrow \mathbf{0}} \int \frac{\mathrm{d}^{2} p_{\perp}}{(2 \pi)^{2}} \operatorname{tr}\left[\gamma^{-} S_{n}^{f}(p+k) \gamma^{+} S_{m}^{f}(p)\right] \\
& =8\left[\left(p_{\|}+k_{\|}\right) \cdot p_{\|}-m_{f}^{2}\right] \frac{\left|q_{f} B\right|}{8 \pi}\left[\theta\left(q_{f} B\right) \delta_{n, m-1}+\theta\left(-q_{f} B\right) \delta_{n-1, m}\right] .
\end{aligned}
$$


From the above expression it is straightforward to read the conductivity. For this purpose to identify the Hall and the transverse conductivities it is useful to express physical quantities in terms of \pm coordinates in accord to $\gamma^{ \pm}$, that is,

$$
j^{ \pm}:=\frac{1}{2}\left(j^{1} \pm \mathrm{i} j^{2}\right), \quad \sigma^{-+}:=\frac{1}{2}\left(\sigma_{\perp}+\mathrm{i} \sigma_{H}\right) .
$$

We can readily confirm that other components (apart from the longitudinal ones) are irrelevant, i.e., $\sigma^{++}=\sigma^{--}=0$. Then, we do not have to compute $\sigma_{H}$ and $\sigma_{\perp}$ individually but we can just separate them from the real part and the imaginary part of $\sigma^{-+}$. We also use a trick to simplify the calculation through the following relation:

$$
\sigma^{-+}=\lim _{k_{0} \rightarrow 0} \lim _{\boldsymbol{k} \rightarrow \mathbf{0}} \frac{1}{2 \mathrm{i} k_{0}}\left[\Pi_{R}^{-+}(k)-\Pi_{A}^{-+}(k)\right]=\lim _{k_{0} \rightarrow 0} \lim _{\boldsymbol{k} \rightarrow \mathbf{0}} \frac{1}{\mathrm{i} k_{0}} \Pi_{R}^{-+}(k) .
$$

This relation holds in our present calculation, but may not be exact in general. In the present case, thanks to the above relation, we can simplify the algebra and little more calculational steps eventually lead us to

$$
\begin{aligned}
\sigma^{-+}= & \lim _{k_{0} \rightarrow 0} \lim _{\boldsymbol{k} \rightarrow \mathbf{0}} \frac{1}{\mathrm{i} k_{0}} \Pi_{R}^{-+}(k) \\
= & N_{\mathrm{c}} \sum_{f} q_{f}^{2} \lim _{k_{0} \rightarrow 0} \lim _{k_{z} \rightarrow 0} \frac{1}{\mathrm{i} k_{0}} \sum_{n, m} \int \frac{\mathrm{d}^{4} p}{(2 \pi)^{4}} \operatorname{tr}\left[\gamma^{-} S_{n}^{f}(k+p) \gamma^{+} S_{m}^{f}(p)\right] \\
& \times\left\{\left[\frac{1}{2}-n_{F}\left(p_{0}+k_{0}-\mu_{f}\right)\right](2 \pi) \operatorname{sgn}\left(p_{0}+k_{0}\right) \delta\left(\left(p_{\|}+k_{\|}\right)^{2}-m_{f n}^{2}\right) \frac{1}{p_{\|}^{2}-m_{f m}^{2}-\mathrm{i} \epsilon p_{0}}\right. \\
& \left.+\frac{1}{\left(p_{\|}+k_{\|}\right)^{2}-m_{f n}^{2}+\mathrm{i} \epsilon\left(p_{0}+k_{0}\right)}\left[\frac{1}{2}-n_{F}\left(p_{0}-\mu_{f}\right)\right](2 \pi) \operatorname{sgn}\left(p_{0}\right) \delta\left(p_{\|}^{2}-m_{f m}^{2}\right)\right\} \\
= & -\mathrm{i} N_{\mathrm{c}} \sum_{f} \operatorname{sgn}\left(q_{f} B\right) \frac{q_{f}^{2}}{2 \pi} \sum_{n} \alpha_{n} \int \frac{\mathrm{d}^{2} p_{\|}}{(2 \pi)^{2}} p_{0}\left[\frac{1}{2}-n_{F}\left(p_{0}-\mu_{f}\right)\right](2 \pi) \operatorname{sgn}\left(p_{0}\right) \delta\left(p_{\|}^{2}-m_{f n}^{2}\right) \\
= & \mathrm{i} N_{\mathrm{c}} \sum_{f} \operatorname{sgn}\left(q_{f} B\right) \frac{q_{f}^{2}}{4 \pi} \sum_{n} \alpha_{n} \int \frac{\mathrm{d} p_{z}}{2 \pi}\left[n_{F}\left(\varepsilon_{f n}-\mu_{f}\right)-n_{F}\left(\varepsilon_{f n}+\mu_{f}\right)\right]=\frac{\mathrm{i}}{2} \cdot \frac{n_{e}}{B} \cdot(3.23)
\end{aligned}
$$

The real part and the imaginary part result in $\sigma_{\perp}=0$ and $\sigma_{H}=n_{e} / B$, respectively, as advertised in eq. (2.8). To find a nonzero value of $\sigma_{\perp}$ we need to go to the two loop calculation which is beyond our present scope. Here, we just give a parametric estimate, that is,

$$
\frac{\sigma_{\perp}}{T} \sim \frac{g^{2} T^{2}}{|q B|},
$$

which is small in our condition of $\sqrt{|q B|} \gg g T$.

This parametric form can be understood from one self-energy insertion of $\Sigma$ to one of the fermion propagators, i.e., in the two-loop order the left-hand side of eq. (3.19) should be replaced with

$$
\lim _{\boldsymbol{k} \rightarrow \mathbf{0}} \operatorname{tr}\left[\gamma^{-} S_{n}^{f}(p+k) \gamma^{+} S_{m}^{f}(p) \Sigma^{f}(p) S_{l}^{f}(p)\right]
$$




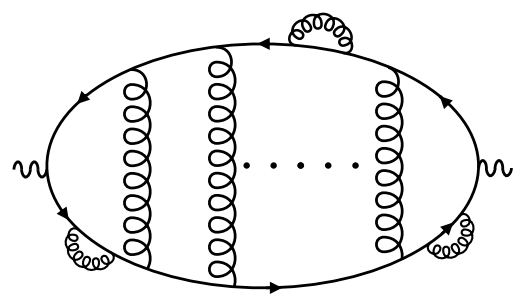

Figure 2. Typical diagram contributing to the leading order calculation of the longitudinal conductivity.
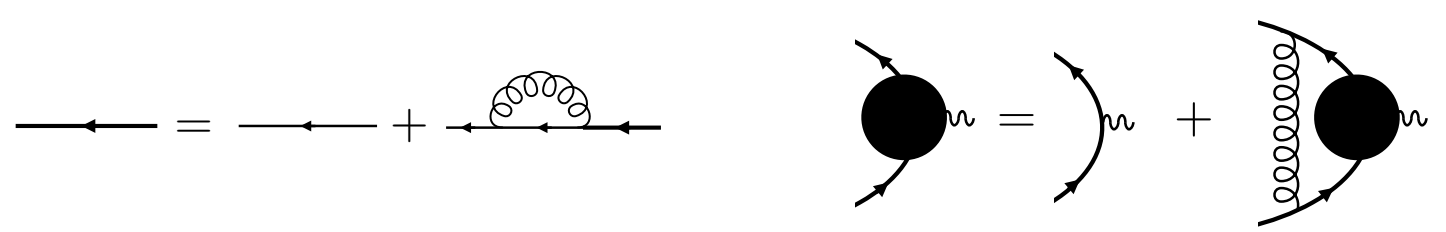

Figure 3. Illustration of the Bethe-Salpeter equations; the resummed propagator with self-energy insertions (left) and the resummed vertex with ladder diagrams (right).

The leading behavior of the self-energy is $\sim g^{2} T^{2}$, while the propagator is of order $1 / \Delta \varepsilon \sim$ $T /|q B|$ where $\Delta \varepsilon$ is an energy gap associated with adjacent Landau levels. Thus, the combination of these factors leads to $\sigma_{\perp} \sim g^{2} T^{2} \cdot T /|q B|=g^{2} T^{2} /|q B|$, which explains eq. (2.9).

\section{Longitudinal conductivity}

This section details the derivation of $\sigma_{\|}$. To this end, we should start with the Kubo formula as we did for $\sigma^{-+}$previously. The calculation for $\sigma_{\|}$is technically involved and we must take account of the resummation of pinching singularities, which is a common technique used also for other transport coefficient calculations (see ref. [47] for example). The pinching singularities generally appear from the following type of integral:

$$
\int \frac{\mathrm{d} k_{0}}{(2 \pi)} F\left(k_{0}\right) \cdot \frac{1}{k_{0}-\varepsilon+\mathrm{i} \gamma} \cdot \frac{1}{k_{0}-\varepsilon-\mathrm{i} \gamma} \sim \frac{F(\varepsilon)}{2 \gamma} .
$$

A typical contribution to the leading order calculation for $\sigma_{\|}$is diagrammatically shown in figure 2. In section 5 we will spell out all field-theoretical transformations, and in this section, let us postpone the derivation and just adopt a kinetic description as a result of the resummation. Although it is nontrivial to prove the equivalence as addressed in section 5 , the kinetic equations provide us with the most efficient approach to evaluate physical observables taking account of the resummation. In fact, in field-theoretical language, the kinetic equations are the Bethe-Salpeter equations, as illustrated schematically in figure 3. We can easily confirm that a diagram like figure 2 is produced by the self-energy insertion and the resummed vertex formulated by iterative processes in figure 3 .

We briefly summarize our strategy to reach $\sigma_{\|}$. We will write down the kinetic or the linearized Boltzmann equations with the collision terms at the lowest scattering order. 
Then, we will compute a distortion, $\delta f$, on the Fermi-Dirac distribution function, $n_{F}$, in response to the applied electric field, which needs an operator inversion on the Boltzmann equations. Once $\delta f$ is worked out in this way, it is easy to represent the electric current in a form proportional to the electric field, and the proportionality coefficient is nothing but the electric conductivity.

\subsection{Boltzmann equations and the formal solution}

The Bethe-Salpeter equations can be translated to the linearized Boltzmann equations with the collision term of scattering processes. The corresponding Boltzmann equations for quarks, anti-quarks, and gluons take the following forms, respectively,

$$
\begin{aligned}
2 P_{p}^{\mu}\left(\partial_{\mu}+q_{f} F_{\nu \mu} \partial_{p_{\nu}}\right) f_{p} & =-C[f], \\
2 \bar{P}_{p^{\prime}}^{\mu}\left(\partial_{\mu}-q_{f} F_{\nu \mu} \partial_{p_{\nu}^{\prime}}\right) \bar{f}_{p^{\prime}} & =-\bar{C}[f], \\
2 k^{\mu} \partial_{\mu} g_{k} & =-\tilde{C}[f],
\end{aligned}
$$

where $\partial_{p_{\nu}}:=\partial / \partial p_{\nu}$ and $C[f], \bar{C}[f]$, and $\tilde{C}[f]$ represent the collision terms. We introduced notations, $2 P_{p}^{\mu}:=\bar{u}(p) \gamma^{\mu} u(p)$ and $2 \bar{P}_{p^{\prime}}^{\mu}:=\bar{v}\left(p^{\prime}\right) \gamma^{\mu} v\left(p^{\prime}\right)$, with the spinor wave functions $u(p)$ and $v\left(p^{\prime}\right)$ for particle and anti-particle, respectively [the precise definition of the wave functions will be given in eq. (5.18)]. For these expressions we use a sloppy notation for the indices; the subscript $p, p^{\prime}$, and $k$ represent not only the momenta but also the Landau level $n$, the angular momentum $l$, the spin $s$, the color $c$, and the flavor $f$.

To solve the Boltzmann equation perturbatively, we expand the distribution functions around the thermal equilibrium, that is,

$$
f_{p}=f_{\mathrm{eq}}(p)+\delta f_{p}, \quad \bar{f}_{p^{\prime}}=\bar{f}_{\mathrm{eq}}\left(p^{\prime}\right)+\delta \bar{f}_{p^{\prime}}, \quad g_{k}=g_{\mathrm{eq}}(k)+\delta g_{k}
$$

with $f_{\text {eq }}(p):=n_{F}\left(\varepsilon_{f n}-\mu_{f}\right), \bar{f}_{\text {eq }}(p):=n_{F}\left(\varepsilon_{f n}+\mu_{f}\right)$, and $g_{\text {eq }}(k):=n_{B}\left(\omega_{k}\right)$ at the local rest frame, where $\omega_{k}=|\boldsymbol{k}|$ is the energy of gluons. In the present problem we are interested in the longitudinal conductivity, so we can consider a homogeneous electric field $E_{z}$ along the $z$ axis up to the linear order in terms of $E_{z}$ and $\delta f_{p}, \delta \bar{f}_{p^{\prime}}, \delta g_{k} \propto E_{z}$. We note that we consider only the "diagonal components" of the distribution functions in spin space, but for our purpose especially without the axial charge coupling, this treatment is sufficient as long as our computation is closed in the linear response regime. For some recent discussions on the full spin dependent distribution functions, see refs. [48-51].

Let us first look at the left-hand side of the Boltzmann equations (4.2). Since the derivative and the field strength are already of order of $E_{z}$, we can safely drop higher order terms involving $\delta f_{p}, \delta \bar{f}_{p^{\prime}}, \delta g_{k}$ and substitute $f_{\text {eq }}(p), \bar{f}_{\text {eq }}\left(p^{\prime}\right)$, and $g_{\text {eq }}(k)$ for $f_{p}, \bar{f}_{p^{\prime}}$, and $g_{k}$. Our assumption of homogeneity makes the spatial derivatives vanishing, and we eventually find,

$$
\begin{aligned}
2 P_{p}^{0}\left(\partial_{0}+q_{f} E_{z} \partial_{p_{z}}\right) f_{\mathrm{eq}}(p) & =-\beta W_{p}\left(-p_{z} \partial_{0} u_{z}+q_{f} E_{z} \frac{p_{z}}{\varepsilon_{f n}}\right), \\
2 P_{p^{\prime}}^{0}\left(\partial_{0}-q_{f} E_{z} \partial_{p_{z}^{\prime}}\right) \bar{f}_{\mathrm{eq}}\left(p^{\prime}\right) & =-\beta \bar{W}_{p^{\prime}}\left(-p_{z}^{\prime} \partial_{0} u_{z}-q_{f} E_{z} \frac{p_{z}^{\prime}}{\varepsilon_{f n^{\prime}}}\right), \\
2 \omega_{k} \partial_{0} g_{\mathrm{eq}}(k) & =-\beta \tilde{W}_{k}\left(-k_{z} \partial_{0} u_{z}\right),
\end{aligned}
$$


where we introduced several new notations. We factorize the derivatives of the thermal distribution functions by introducing the following functions:

$$
\begin{aligned}
W_{p} & :=2 P_{p}^{0} f_{\mathrm{eq}}(p)\left[1-f_{\mathrm{eq}}(p)\right], \\
\bar{W}_{p^{\prime}} & :=2 P_{p^{\prime}}^{0} \bar{f}_{\mathrm{eq}}\left(p^{\prime}\right)\left[1-\bar{f}_{\mathrm{eq}}\left(p^{\prime}\right)\right], \\
\tilde{W}_{k} & :=2 \omega_{k} g_{\mathrm{eq}}(k)\left[1+g_{\mathrm{eq}}(k)\right],
\end{aligned}
$$

which will be used as the weight functions in the inner product in later calculations. The first terms appear from time-dependent fluid velocity $u_{z}$ which is induced by the $E_{z}$ effect. We note that in the linear response regime no fluid velocity is developed yet, but $\partial_{0} u_{z}$ can be nonvanishing. One can easily understand how $\partial_{0} u_{z}$ terms emerge by replacing $\varepsilon_{f n} \rightarrow p_{f n} \cdot u$ and $\omega_{k} \rightarrow k \cdot u$ with the fluid velocity $u^{\mu}$. Now, we should quantify $\partial_{0} u_{z}$ in response to $E_{z}$; for this purpose we can use the leading order hydrodynamic equation, $\partial_{0} u_{z}=n_{e} E_{z} /\left(\mathcal{E}+\mathcal{P}_{z}\right)$, which can be immediately understood from $\partial_{\mu} T^{\mu \nu}=F_{\mu}^{\nu} j_{\mathrm{em}}^{\mu}$. Here, as mentioned below eq. (2.4), $\mathcal{E}$ is the energy density and $\mathcal{P}_{z}$ is the pressure in the $z$ direction, which are explicitly given, respectively, as

$$
\begin{aligned}
\mathcal{E} & :=N_{\mathrm{c}} \sum_{f, n} \alpha_{n} \frac{\left|q_{f} B\right|}{2 \pi} \int \frac{\mathrm{d} p_{z}}{2 \pi} \varepsilon_{f n}\left[f_{\mathrm{eq}}(p)+\bar{f}_{\mathrm{eq}}(p)\right]+2\left(N_{\mathrm{c}}^{2}-1\right) \int \frac{\mathrm{d}^{3} k}{(2 \pi)^{3}} \omega_{k} g_{\mathrm{eq}}(k), \\
\mathcal{P}_{z} & :=N_{\mathrm{c}} \sum_{f, n} \alpha_{n} \frac{\left|q_{f} B\right|}{2 \pi} \int \frac{\mathrm{d} p_{z}}{2 \pi} \frac{p_{z}^{2}}{\varepsilon_{f n}}\left[f_{\mathrm{eq}}(p)+\bar{f}_{\mathrm{eq}}(p)\right]+2\left(N_{\mathrm{c}}^{2}-1\right) \int \frac{\mathrm{d}^{3} k}{(2 \pi)^{3}} \frac{k_{z}^{2}}{\omega_{k}} g_{\mathrm{eq}}(k) .
\end{aligned}
$$

We have finished the preparation, and we can now specify the collision integrals to solve $\delta f_{p}, \delta \bar{f}_{p^{\prime}}$, and $\delta g_{k}$. Before doing so, however, let us introduce symbolic representations to further sort out expressions algebraically.

Instead of the original Boltzmann equations (4.2), we write them concisely as

$$
\mathcal{S}=\mathcal{L} \chi
$$

For convenience we move $W_{p}, \bar{W}_{p^{\prime}}$, and $\tilde{W}_{k}$ as well as $E_{z}$ from the left-hand side to the right-hand side, and then we find,

$$
\mathcal{S}:=\mathcal{J}^{z}-\frac{n_{e} \mathcal{T}^{0 z}}{\mathcal{E}+\mathcal{P}_{z}}
$$

from eq. (4.4), where

$$
\mathcal{J}^{\mu}:=\left(\begin{array}{c}
q_{f} p^{\mu} / \varepsilon_{f n} \\
-q_{f} p^{\prime \mu} / \varepsilon_{f n^{\prime}} \\
0
\end{array}\right), \quad \mathcal{T}^{0 \mu}:=\left(\begin{array}{c}
p^{\mu} \\
p^{\prime \mu} \\
k^{\mu}
\end{array}\right)
$$

Interestingly, this form is analogous to the expectation value of the each flavor current with subtraction as in eq. (2.4), which will turn out to be crucial to make the calculation well-defined. The right-hand side of eq. (4.10), with $W_{p}, \bar{W}_{p^{\prime}}, \tilde{W}_{k}$, and $E_{z}$ moved from the 
left-hand side, simply reads,

$$
\mathcal{L} \chi:=\frac{1}{E_{z}}\left(\begin{array}{c}
\frac{1}{\beta W_{p}} C[f] \\
\frac{1}{\beta \bar{W}_{p^{\prime}}} \bar{C}[f] \\
\frac{1}{\beta \tilde{W}_{k}} \tilde{C}[f]
\end{array}\right) .
$$

In the next subsection we will present all the details about the collision terms. For the moment it should be noted that $C=\bar{C}=\tilde{C}=0$ for equilibrium distributions, and so $\mathcal{L} \chi$ should be of order of $\delta f_{p}, \delta \bar{f}_{p^{\prime}}$, and $\delta g_{k}$.

At this point, we shall introduce some more notations which are useful to simplify actual computations. To eliminate kinematic factors in the denominators of eq. (4.13), we rescale $\delta f_{p}, \delta \bar{f}_{p^{\prime}}$, and $\delta g_{k}$ as

$$
\begin{aligned}
\delta f_{p} & =\beta f_{\mathrm{eq}}(p)\left[1-f_{\mathrm{eq}}(p)\right] E_{z} \chi_{p}, \\
\delta \bar{f}_{p^{\prime}} & =\beta \bar{f}_{\mathrm{eq}}\left(p^{\prime}\right)\left[1-\bar{f}_{\mathrm{eq}}\left(p^{\prime}\right)\right] E_{z} \bar{\chi}_{p^{\prime}}, \\
\delta g_{k} & =\beta g_{\mathrm{eq}}(k)\left[1+g_{\mathrm{eq}}(k)\right] E_{z} \tilde{\chi}_{k} .
\end{aligned}
$$

Introducing a vector symbol,

$$
\chi:=\left(\begin{array}{c}
\chi_{p} \\
\bar{\chi}_{p^{\prime}} \\
\tilde{\chi}_{k}
\end{array}\right)
$$

we can interpret eq. (4.13) as a linear operation of $\mathcal{L}$ onto $\chi$, which means that the formal solution, $\chi=\mathcal{L}^{-1} \mathcal{S}$, gives the longitudinal conductivity as follows. We can write down the electric current parallel to $B$ along the $z$ axis and the longitudinal conductivity as

$$
\begin{aligned}
\sigma_{\|} & =\frac{j_{z}}{E_{z}}=N_{\mathrm{c}} \sum_{f} \frac{q_{f}\left|q_{f} B\right|}{2 \pi} \sum_{n=0}^{\infty} \alpha_{n} \int \frac{\mathrm{d} p_{z}}{2 \pi} \frac{p_{z}}{\varepsilon_{f n}}\left(\frac{\delta f_{p}}{E_{z}}-\frac{\delta \bar{f}_{p}}{E_{z}}\right) \\
& =\beta N_{\mathrm{c}} \sum_{f} \frac{q_{f}\left|q_{f} B\right|}{2 \pi} \sum_{n=0}^{\infty} \alpha_{n} \int \frac{\mathrm{d} p_{z}}{2 \pi} \frac{p_{z}}{\varepsilon_{f n}}\left\{f_{\mathrm{eq}}(p)\left[1-f_{\mathrm{eq}}(p)\right] \chi_{p}-\bar{f}_{\mathrm{eq}}(p)\left[1-\bar{f}_{\mathrm{eq}}(p)\right] \bar{\chi}_{p}\right\} .
\end{aligned}
$$

Up to now, all necessary ingredients except for the collision terms have been presented. However, these are not yet adequate for real calculations; $\mathcal{L}$ contains zero eigenvalues with the eigenvectors, $\mathcal{C}^{a}=\left\{\mathcal{J}^{0}, \mathcal{T}^{0 \mu}\right\}$ (i.e., hydrodynamic modes), corresponding to the charge and the energy-momentum conservations. Here, we suppress the flavor index below to avoid cumbersome notations.

To make $\mathcal{L}^{-1}$ well-defined, we should get rid of such zero modes. We must emphasize that this removal of zero modes is not an ad hoc procedure; $\mathcal{S}=\mathcal{L} \chi$ is a perfectly welldefined equation to solve $\chi$ because of the special structure of $\mathcal{S}$. To see this point in a clear way, our symbolic representation is useful. 
Since we want to discuss the projection operation, we need to define an inner product. A natural choice of the inner product of two functions, $A=\left(a_{p}, \bar{a}_{p^{\prime}}, \tilde{a}_{k}\right)$ and $B=\left(b_{p}, \bar{b}_{p^{\prime}}, \tilde{b}_{k}\right)$, should be

$$
(A, B):=\int_{p} W_{p} a_{p} b_{p}+\int_{p^{\prime}} \bar{W}_{p^{\prime}} \bar{a}_{p^{\prime}} \bar{b}_{p^{\prime}}+\int_{k} \tilde{W}_{k} \tilde{a}_{k} \tilde{b}_{k}
$$

where we used a simplified notation for all the phase space sum,

$$
\int_{p}:=\sum_{n, l, c, s, f} \int \frac{\mathrm{d}^{3} p}{(2 \pi)^{3}} \frac{1}{2 \varepsilon_{f n}}, \quad \int_{k}:=\sum_{c, s} \int \frac{\mathrm{d}^{3} k}{(2 \pi)^{3}} \frac{1}{2 \omega_{k}} .
$$

With this definition of the inner product the longitudinal electric conductivity (4.16) takes an extremely simple form as

$$
\sigma_{\|}=\beta\left(\mathcal{J}^{z}, \chi\right)
$$

from an explicit expression of $\mathcal{J}^{z}$ in eq. (4.12). Now, using the zero eigenvector $\mathcal{C}$ and the inner product as defined above, we introduce a projection operator $\mathcal{Q}$ onto functional space excluding zero eigenvalues as

$$
\mathcal{Q} O:=O-\sum_{a, b} \mathcal{C}^{a}(\mathcal{C}, \mathcal{C})_{a b}^{-1}\left(\mathcal{C}^{b}, O\right)
$$

where $(\mathcal{C}, \mathcal{C})_{a b}^{-1}$ is the inverse matrix of $\left(\mathcal{C}^{a}, \mathcal{C}^{b}\right)$. We immediately see $\mathcal{Q}^{2}=\mathcal{Q}$ and $\mathcal{Q C}^{a}=0$ by construction. Using an alternative expression of the charge density and the enthalpy, $n_{e}=\beta\left(\mathcal{T}^{0 z}, \mathcal{J}^{z}\right)$ and $\mathcal{E}+\mathcal{P}_{z}=\beta\left(\mathcal{T}^{0 z}, \mathcal{T}^{0 z}\right)$ [52], we can rewrite eq. (4.11) into $\mathcal{S}=\mathcal{Q} \mathcal{J}^{z}$. Because zero eigenvalues simply give zero, $\mathcal{L}=\mathcal{L Q}$ trivially follows, and the equation, $\mathcal{L} \chi=\mathcal{S}$, is equivalent to $\mathcal{Q} \mathcal{L} \chi=\mathcal{Q S}=\mathcal{S}$, which can be solved as $\chi=\mathcal{Q} \mathcal{L}^{-1} \mathcal{Q S}$. We eventually obtain,

$$
\sigma_{\|}=\beta\left(\mathcal{J}^{z}, \mathcal{Q} \mathcal{L}^{-1} \mathcal{Q S}\right)=\beta\left(\mathcal{S}, \mathcal{L}^{-1} \mathcal{S}\right)=\beta(\mathcal{S}, \chi)
$$

\subsection{Collision terms}

The last missing pieces are the collision terms, $C[f], \bar{C}[f]$, and $\tilde{C}[f]$. In this work we consider the weak coupling expansion with $g^{2} \ll 1$, and the lowest order contributions then arise from $1 \leftrightarrow 2$ processes. We note that the typical scale of $1 \leftrightarrow 2$ processes is $\sim g^{2} q_{f} B / T^{2}$ which is much larger than the typical scale $\sim g^{4}$ of $2 \leftrightarrow 2$ processes under our hierarchy (2.7).

Such $1 \leftrightarrow 2$ processes can be decomposed as

$$
\begin{aligned}
C[f] & =C_{q \rightarrow q g}[f]+C_{q g \rightarrow q}[f]+C_{q \bar{q} \rightarrow g}[f], \\
\bar{C}[f] & =\bar{C}_{\bar{q} \rightarrow \bar{q} g}[f]+\bar{C}_{\bar{q} g \rightarrow \bar{q}}[f]+\bar{C}_{q \bar{q} \rightarrow g}[f], \\
\tilde{C}[f] & =\tilde{C}_{g \rightarrow q \bar{q}}[f]+\tilde{C}_{q g \rightarrow q}[f]+\tilde{C}_{\bar{q} g \rightarrow \bar{q}}[f],
\end{aligned}
$$

where the subscripts represent $1 \leftrightarrow 2$ processes as illustrated in figure 4 . Once the scattering amplitude $\mathcal{M}$ is given (which we will compute later), the kinematical assignments of the 


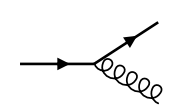

(a1) $q \rightarrow q g$

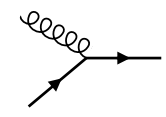

(a2) $q g \rightarrow q$

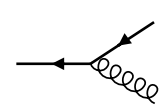

(b1) $\bar{q} \rightarrow \bar{q} g$

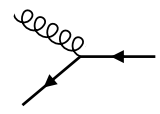

(b2) $\bar{q} g \rightarrow \bar{q}$

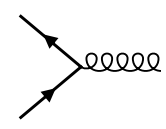

(c1) $q \bar{q} \rightarrow g$

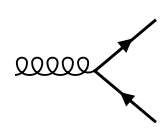

(c2) $g \rightarrow q \bar{q}$

Figure 4. Diagrams of the radiation process with a quark (a1) and a anti-quark (b1), the pair annihilation (c1), and their inverse processes, (a2), (b2), and (c2).

distribution functions lead to the following expressions:

$$
\begin{aligned}
& C_{q \rightarrow q g}[f]=\int_{k, p^{\prime}}\left|\mathcal{M}_{p \rightarrow p^{\prime}+k}\right|^{2}(2 \pi)^{4} \delta^{(4)}\left(p-k-p^{\prime}\right)\left[f_{p}\left(1-f_{p^{\prime}}\right)\left(1+g_{k}\right)-\left(1-f_{p}\right) f_{p^{\prime}} g_{k}\right], \\
& C_{q g \rightarrow q}[f]=\int_{k, p^{\prime}}\left|\mathcal{M}_{p+k \rightarrow p^{\prime}}\right|^{2}(2 \pi)^{4} \delta^{(4)}\left(p+k-p^{\prime}\right)\left[f_{p} g_{k}\left(1-f_{p^{\prime}}\right)-\left(1-f_{p}\right)\left(1+g_{k}\right) f_{p^{\prime}}\right], \\
& C_{q \bar{q} \rightarrow g}[f]=\int_{k, p^{\prime}}\left|\mathcal{M}_{p+p^{\prime} \rightarrow k}\right|^{2}(2 \pi)^{4} \delta^{(4)}\left(p+p^{\prime}-k\right)\left[f_{p} \bar{f}_{p^{\prime}}\left(1+g_{k}\right)-\left(1-f_{p}\right)\left(1-\bar{f}_{p^{\prime}}\right) g_{k}\right]
\end{aligned}
$$

for quarks and

$$
\begin{aligned}
& \bar{C}_{\bar{q} \rightarrow \bar{q} g}[f]=\int_{k, p^{\prime}}\left|\mathcal{M}_{p \rightarrow p^{\prime}+k}\right|^{2}(2 \pi)^{4} \delta^{(4)}\left(p-k-p^{\prime}\right)\left[\bar{f}_{p}\left(1-\bar{f}_{p^{\prime}}\right)\left(1+g_{k}\right)-\left(1-\bar{f}_{p}\right) \bar{f}_{p^{\prime}} g_{k}\right], \\
& \bar{C}_{\bar{q} g \rightarrow \bar{q}}[f]=\int_{k, p^{\prime}}\left|\mathcal{M}_{p+k \rightarrow p^{\prime}}\right|^{2}(2 \pi)^{4} \delta^{(4)}\left(p+k-p^{\prime}\right)\left[\bar{f}_{p} g_{k}\left(1-\bar{f}_{p^{\prime}}\right)-\left(1-\bar{f}_{p}\right)\left(1+g_{k}\right) \bar{f}_{p^{\prime}}\right], \\
& \bar{C}_{q \bar{q} \rightarrow g}[f]=\int_{k, p^{\prime}}\left|\mathcal{M}_{p+p^{\prime} \rightarrow k}\right|^{2}(2 \pi)^{4} \delta^{(4)}\left(p+p^{\prime}-k\right)\left[\bar{f}_{p} f_{p^{\prime}}\left(1+g_{k}\right)-\left(1-\bar{f}_{p}\right)\left(1-f_{p^{\prime}}\right) g_{k}\right]
\end{aligned}
$$

for anti-quarks and

$$
\begin{aligned}
& \tilde{C}_{g \rightarrow q \bar{q}}[f]=\int_{p, p^{\prime}}\left|\mathcal{M}_{k \rightarrow p+p^{\prime}}\right|^{2}(2 \pi)^{4} \delta^{(4)}\left(k-p-p^{\prime}\right)\left[g_{k}\left(1-f_{p}\right)\left(1-\bar{f}_{p^{\prime}}\right)-\left(1+g_{k}\right) f_{p} \bar{f}_{p^{\prime}}\right], \\
& \tilde{C}_{q g \rightarrow q}[f]=\int_{p, p^{\prime}}\left|\mathcal{M}_{k+p \rightarrow p^{\prime}}\right|^{2}(2 \pi)^{4} \delta^{(4)}\left(k+p-p^{\prime}\right)\left[g_{k} f_{p}\left(1-f_{p^{\prime}}\right)-\left(1+g_{k}\right)\left(1-f_{p}\right) f_{p^{\prime}}\right], \\
& \tilde{C}_{\bar{q} g \rightarrow \bar{q}}[f]=\int_{p, p^{\prime}}\left|\mathcal{M}_{k+p^{\prime} \rightarrow p}\right|^{2}(2 \pi)^{4} \delta^{(4)}\left(k+p^{\prime}-p\right)\left[g_{k} \bar{f}_{p^{\prime}}\left(1-\bar{f}_{p}\right)-\left(1+g_{k}\right) \bar{f}_{p}\left(1-\bar{f}_{p^{\prime}}\right)\right]
\end{aligned}
$$

for gluons. Here, $f_{p}, \bar{f}_{p^{\prime}}$, and $g_{k}$ represent the quark, the anti-quark, and the gluon distribution functions (i.e., the thermal equilibrium distributions plus fluctuations) with quantum numbers $p, p^{\prime}$, and $k$, respectively. Then, the collision terms are vanishing with $f_{\text {eq }}(p)$, $\bar{f}_{\text {eq }}\left(p^{\prime}\right)$, and $g_{\text {eq }}(k)$ due to the detailed balance, and the first nonvanishing terms are linear in terms of $\delta f_{p}, \delta \bar{f}_{p^{\prime}}$, and $\delta g_{k}$ (or $\chi_{p}, \bar{\chi}_{p^{\prime}}$, and $\tilde{\chi}_{k}$ ). Simple calculations lead us to

$$
\begin{aligned}
\frac{C[f]}{\beta E_{z}}= & \int_{k, p^{\prime}}\left|\mathcal{M}_{p \rightarrow p^{\prime}+k}\right|^{2}(2 \pi)^{4} \delta^{(4)}\left(p-k-p^{\prime}\right) f_{\mathrm{eq}}(p)\left[1-f_{\mathrm{eq}}\left(p^{\prime}\right)\right]\left[1+g_{\mathrm{eq}}(k)\right]\left(\chi_{p}-\tilde{\chi}_{k}-\chi_{p^{\prime}}\right) \\
& +\int_{k, p^{\prime}}\left|\mathcal{M}_{p+k \rightarrow p^{\prime}}\right|^{2}(2 \pi)^{4} \delta^{(4)}\left(p+k-p^{\prime}\right) f_{\mathrm{eq}}(p) g_{\mathrm{eq}}(k)\left[1-f_{\mathrm{eq}}\left(p^{\prime}\right)\right]\left(\chi_{p}+\tilde{\chi}_{k}-\chi_{p^{\prime}}\right) \\
& +\int_{k, p^{\prime}}\left|\mathcal{M}_{p+p^{\prime} \rightarrow k}\right|^{2}(2 \pi)^{4} \delta^{(4)}\left(p+p^{\prime}-k\right) f_{\mathrm{eq}}(p) \bar{f}_{\mathrm{eq}}\left(p^{\prime}\right)\left[1+g_{\mathrm{eq}}(k)\right]\left(\chi_{p}+\bar{\chi}_{p^{\prime}}-\tilde{\chi}_{k}\right)
\end{aligned}
$$


for quarks and

$$
\begin{aligned}
\frac{\bar{C}[f]}{\beta E_{z}}= & \int_{k, p^{\prime}}\left|\mathcal{M}_{p \rightarrow p^{\prime}+k}\right|^{2}(2 \pi)^{4} \delta^{(4)}\left(p-k-p^{\prime}\right) \bar{f}_{\mathrm{eq}}(p)\left[1-\bar{f}_{\mathrm{eq}}\left(p^{\prime}\right)\right]\left[1+g_{\mathrm{eq}}(k)\right]\left(\bar{\chi}_{p}-\tilde{\chi}_{k}-\bar{\chi}_{p^{\prime}}\right) \\
& +\int_{k, p^{\prime}}\left|\mathcal{M}_{p+k \rightarrow p^{\prime}}\right|^{2}(2 \pi)^{4} \delta^{(4)}\left(p+k-p^{\prime}\right) \bar{f}_{\mathrm{eq}}(p) g_{\mathrm{eq}}(k)\left[1-\bar{f}_{\mathrm{eq}}\left(p^{\prime}\right)\right]\left(\bar{\chi}_{p}+\tilde{\chi}_{k}-\bar{\chi}_{p^{\prime}}\right) \\
& +\int_{k, p^{\prime}}\left|\mathcal{M}_{p+p^{\prime} \rightarrow k}\right|^{2}(2 \pi)^{4} \delta^{(4)}\left(p+p^{\prime}-k\right) \bar{f}_{\mathrm{eq}}(p) f_{\mathrm{eq}}\left(p^{\prime}\right)\left[1+g_{\mathrm{eq}}(k)\right]\left(\bar{\chi}_{p}+\chi_{p^{\prime}}-\tilde{\chi}_{k}\right)
\end{aligned}
$$

for anti-quarks, and

$$
\begin{aligned}
\frac{\tilde{C}[f]}{\beta E_{z}}= & \int_{p, p^{\prime}}\left|\mathcal{M}_{k \rightarrow p+p^{\prime}}\right|^{2}(2 \pi)^{4} \delta^{(4)}\left(k-p-p^{\prime}\right) g_{\mathrm{eq}}(k)\left[1-f_{\mathrm{eq}}(p)\right]\left[1-\bar{f}_{\mathrm{eq}}\left(p^{\prime}\right)\right]\left(\tilde{\chi}_{k}-\chi_{p}-\bar{\chi}_{p^{\prime}}\right) \\
& +\int_{p, p^{\prime}}\left|\mathcal{M}_{k+p \rightarrow p^{\prime}}\right|^{2}(2 \pi)^{4} \delta^{(4)}\left(k+p-p^{\prime}\right) g_{\mathrm{eq}}(k) f_{\mathrm{eq}}(p)\left[1-f_{\mathrm{eq}}\left(p^{\prime}\right)\right]\left(\tilde{\chi}_{k}+\chi_{p}-\chi_{p^{\prime}}\right) \\
& +\int_{p, p^{\prime}}\left|\mathcal{M}_{k+p^{\prime} \rightarrow p}\right|^{2}(2 \pi)^{4} \delta^{(4)}\left(k+p^{\prime}-p\right) g_{\mathrm{eq}}(k) \bar{f}_{\mathrm{eq}}\left(p^{\prime}\right)\left[1-\bar{f}_{\mathrm{eq}}(p)\right]\left(\tilde{\chi}_{k}+\bar{\chi}_{p^{\prime}}-\bar{\chi}_{p}\right)
\end{aligned}
$$

for gluons.

Now our problem is reduced to the calculation of the scattering amplitudes. It is straightforward to write down the amplitude contributions from the synchrotron radiation and the pair annihilation processes as follows:

$$
\begin{aligned}
& \mathrm{i} \mathcal{M}_{p \rightarrow k+p^{\prime}}=\mathrm{i} g \bar{u}\left(p^{\prime}\right) \gamma^{\mu} t_{a} u(p) \varepsilon_{\mu}^{*}(s, k), \\
& \mathrm{i} \mathcal{M}_{p+p^{\prime} \rightarrow k}=\mathrm{i} g \bar{v}\left(p^{\prime}\right) \gamma^{\mu} t_{a} u(p) \varepsilon_{\mu}^{*}(s, k) .
\end{aligned}
$$

We can make the sum of the squared amplitudes over the spin $s$, the angular momentum $l$, and the color $c$ to find expressions in terms of the Dirac structures (3.6) of the propagator, i.e.,

$$
\begin{aligned}
& \sum_{s, l, c}\left|\mathcal{M}_{p \rightarrow k+p^{\prime}}\right|^{2}=-\delta_{f f^{\prime}} g^{2} N_{\mathrm{c}} C_{F} \operatorname{tr}\left[\gamma_{\mu} S_{n}^{f}(p) \gamma^{\mu} S_{n^{\prime}}^{f}(p-k)\right], \\
& \sum_{s, l, c}\left|\mathcal{M}_{p+p^{\prime} \rightarrow k}\right|^{2}=+\delta_{f f^{\prime}} g^{2} N_{\mathrm{c}} C_{F} \operatorname{tr}\left[\gamma_{\mu} S_{n}^{f}(p) \gamma^{\mu} S_{n^{\prime}}^{f}(p-k)\right],
\end{aligned}
$$

where the color factor is $C_{F}=\left(N_{\mathrm{c}}^{2}-1\right) /\left(2 N_{\mathrm{c}}\right)$ and $p$ and $k$ in the above expression refer to the momenta only. We replaced the polarization sum $\sum_{s} \varepsilon_{\mu}^{*}(s, k) \varepsilon_{\nu}(s, k)$ by $-\eta_{\mu \nu}$ thanks to the gauge invariance of the scattering amplitude, and transformed the Dirac structures using $\sum_{l, s} u(p) \bar{u}(p)=S_{n}^{f}(p)$ and $\sum_{l, s} v(p) \bar{v}(p)=-S_{n}^{f}(-p)$.

One might think that the amplitudes for the synchrotron radiation and the pair annihilation process may look identical from eqs. (4.37) and (4.38) apart from the overall sign, but the kinematic regions of involved momenta are quite different. For the synchrotron radiation process of eq. (4.37) with $p \rightarrow k+p^{\prime}$, the on-shell condition reads, $\varepsilon_{f n}-\omega_{k}-\varepsilon_{f n^{\prime}}=0$ 
(which is possible for $n>n^{\prime}$ ), from which we can solve $\xi_{k}^{f}=\left|\boldsymbol{k}_{\perp}\right|^{2} /\left(2\left|q_{f} B\right|\right.$ ), that is given as

$$
\xi_{k}^{f}=\xi_{-}^{f}=\frac{\left(\varepsilon_{f n}-\varepsilon_{f n^{\prime}}\right)^{2}-\left(p_{z}-p_{z}^{\prime}\right)^{2}}{2\left|q_{f} B\right|} .
$$

This quantity should be non-negative from the definition of $\xi_{k}^{f}$, so that the integration range of $p_{z}$ and $p_{z}^{\prime}$ should be restricted to satisfy,

$$
m_{f n}^{2} p_{z}^{\prime 2}+m_{f n^{\prime}}^{2} p_{z}^{2}-p_{z} p_{z}^{\prime}\left(m_{f n}^{2}+m_{f n^{\prime}}^{2}\right)-\frac{1}{4}\left(m_{f n}^{2}-m_{f n^{\prime}}^{2}\right)^{2} \leq 0 .
$$

For given $p_{z}$, equivalently, we find that $p_{z}^{\prime}$ ranges in $p_{z-}^{\prime} \leq p_{z}^{\prime} \leq p_{z+}^{\prime}$, where

$$
p_{z \pm}^{\prime}=p_{z} \frac{m_{f n}^{2}+m_{f n^{\prime}}^{2}}{2 m_{f n}^{2}} \pm \frac{m_{f n}^{2}-m_{f n^{\prime}}^{2}}{2 m_{f n}^{2}} \sqrt{m_{f n}^{2}+p_{z}^{2}} .
$$

Thus, the phase space integration for the synchrotron radiation process is rewritten as

$$
\begin{aligned}
& \int \frac{\mathrm{d}^{3} k}{(2 \pi)^{3}} \frac{1}{2 \omega_{k}}(2 \pi) \delta\left(\omega_{k}-\varepsilon_{f n}+\varepsilon_{f n^{\prime}}\right) \cdots \\
& =\frac{1}{2} \vartheta\left(n-n^{\prime}-1\right) \int \frac{\mathrm{d} k_{z}}{2 \pi} \int \mathrm{d} \xi_{k}^{f} \delta\left(\xi_{k}^{f}-\xi_{-}^{f}\right) \theta\left(p_{z+}^{\prime}-p_{z}^{\prime}\right) \theta\left(p_{z}^{\prime}-p_{z-}^{\prime}\right) \cdots,
\end{aligned}
$$

where $\vartheta(x)$ is the unit step function,

$$
\vartheta(x):=\left\{\begin{array}{ll}
0 & (x<0) \\
1 & (x \geq 0)
\end{array} .\right.
$$

In the same way, we can identify the phase space for the pair annihilation process of eq. (4.38) with $p+p^{\prime} \rightarrow k$. From the energy on-shell condition, $\xi_{k}^{f}$ is fixed as

$$
\xi_{k}^{f}=\xi_{+}^{f}=\frac{\left(\varepsilon_{f n}+\varepsilon_{f n^{\prime}}\right)^{2}-\left(p_{z}+p_{z}^{\prime}\right)^{2}}{2\left|q_{f} B\right|} .
$$

It is easy to confirm that $\xi_{+}^{f}$ is positive definite, and so the integrations of $p_{z}$ and $p_{z}^{\prime}$ are not limited unlike the synchrotron radiation. Then, the phase space integration for the pair annihilation process should be

$$
\int \frac{\mathrm{d}^{3} k}{(2 \pi)^{3}} \frac{1}{2 \omega_{k}}(2 \pi) \delta\left(\omega_{k}-\varepsilon_{f n}-\varepsilon_{f n^{\prime}}\right) \cdots=\frac{1}{2} \int \frac{\mathrm{d} k_{z}}{2 \pi} \int \mathrm{d} \xi_{k}^{f} \delta\left(\xi_{k}^{f}-\xi_{+}^{f}\right) \cdots .
$$

These assignments of allowed kinematic regions lead us to the following distinctions in the collision terms as

$$
\begin{aligned}
& \int_{k, p, p^{\prime}}\left|\mathcal{M}_{p \rightarrow p^{\prime}+k}\right|^{2}(2 \pi)^{4} \delta^{(4)}\left(k-p+p^{\prime}\right)=-\frac{1}{2} \sum_{f, n>n^{\prime}} \int \frac{\mathrm{d} p_{z}}{2 \pi} \frac{1}{2 \varepsilon_{f n}} \int^{\prime} \frac{\mathrm{d} p_{z}^{\prime}}{2 \pi} \frac{1}{2 \varepsilon_{f n^{\prime}}} X\left(n, n^{\prime}, \xi_{-}\right), \\
& \int_{k, p, p^{\prime}}\left|\mathcal{M}_{p+p^{\prime} \rightarrow k}\right|^{2}(2 \pi)^{4} \delta^{(4)}\left(p+p^{\prime}-k\right)=\frac{1}{2} \sum_{f, n, n^{\prime}} \int \frac{\mathrm{d} p_{z}}{2 \pi} \frac{1}{2 \varepsilon_{f n}} \int \frac{\mathrm{d} p_{z}^{\prime}}{2 \pi} \frac{1}{2 \varepsilon_{f n^{\prime}}} X\left(n, n^{\prime}, \xi_{+}\right),
\end{aligned}
$$


where the common integrand is given from eqs. (4.37) and (4.38) as

$$
X\left(n, n^{\prime}, \xi_{k}\right):=g^{2} N_{\mathrm{c}} C_{F} \int \frac{\mathrm{d}^{2} p_{\perp}}{(2 \pi)^{2}} \operatorname{tr}\left[\gamma_{\mu} S_{n}^{f}(p) \gamma^{\mu} S_{n^{\prime}}^{f}(p-k)\right] .
$$

Here, in eq. (4.46), we introduced a compact notation for the momentum integration as

$$
\int^{\prime} \frac{\mathrm{d} p_{z}^{\prime}}{2 \pi}:=\int_{p_{z-}^{\prime}}^{p_{z+}^{\prime}} \frac{\mathrm{d} p_{z}^{\prime}}{2 \pi}
$$

using $p_{z \pm}^{\prime}$ defined in eq. (4.41). Because the final form of $X\left(n, n^{\prime}, \xi\right)$ is complicated, let us first give the final form here, and then look at major steps of the derivation. The final expression reads,

$$
\begin{aligned}
X\left(n, n^{\prime}, \xi\right)= & g^{2} N_{\mathrm{c}} C_{F} \frac{\left|q_{f} B\right|}{2 \pi} \mathrm{e}^{-\xi} \frac{n !}{n^{\prime} !} \xi^{n^{\prime}-n}\left\{\left[4 m_{f}^{2}-4\left|q_{f} B\right|\left(n+n^{\prime}-\xi\right) \frac{1}{\xi}\left(n+n^{\prime}\right)\right] F\left(n, n^{\prime}, \xi\right)\right. \\
& \left.+16\left|q_{f} B\right| n^{\prime}\left(n+n^{\prime}\right) \frac{1}{\xi} L_{n}^{\left(n^{\prime}-n\right)}(\xi) L_{n-1}^{\left(n^{\prime}-n\right)}(\xi)\right\},
\end{aligned}
$$

where we introduced a new function, $F\left(n, n^{\prime}, \xi\right)$, defined by

$$
F\left(n, n^{\prime}, \xi\right):= \begin{cases}1 & (n=0) \\ {\left[L_{n}^{\left(n^{\prime}-n\right)}(\xi)\right]^{2}+\frac{n^{\prime}}{n}\left[L_{n-1}^{\left(n^{\prime}-n\right)}(\xi)\right]^{2}} & (n>0) .\end{cases}
$$

All calculational details would be quite lengthy, but we shall give a sketch of how to arrive at the above final form from eqs. (4.37) and (4.38) so that interested readers could reproduce it. Using eq. (3.6) a common building block of eqs. (4.37) and (4.38) is expressed as

$$
\gamma_{\mu} S_{n}^{f}(p) \gamma^{\mu}=2\left[\left(-P_{-}^{f} \not p_{\|}+m_{f}\right) A_{n+}+\left(-P_{+}^{f} \not p_{\|}+m_{f}\right) A_{n-}-\not p_{\perp} B_{n}\right]
$$

Taking the Dirac trace in eqs. (4.37) and (4.38) is straightforward, which yields,

$$
\begin{aligned}
\operatorname{tr} & {\left[\gamma_{\mu} S_{n}^{f}(p) \gamma^{\mu} S_{n^{\prime}}^{f}(p-k)\right] } \\
= & 4 m_{f}^{2}\left[A_{n^{\prime}+}\left(4 \xi_{p-k}^{f}\right) A_{n+}\left(4 \xi_{p}^{f}\right)+A_{n^{\prime}-}\left(4 \xi_{p-k}^{f}\right) A_{n-}\left(4 \xi_{p}^{f}\right)\right] \\
& -4\left[\left(p_{\|}-k_{\|}\right) \cdot p_{\|}-m_{f}^{2}\right]\left[A_{n^{\prime}-}\left(4 \xi_{p-k}^{f}\right) A_{n+}\left(4 \xi_{p}^{f}\right)+A_{n^{\prime}+}\left(4 \xi_{p-k}^{f}\right) A_{n-}\left(4 \xi_{p}^{f}\right)\right] \\
& +8\left(\boldsymbol{p}_{\perp}-\boldsymbol{k}_{\perp}\right) \cdot \boldsymbol{p}_{\perp} B_{n^{\prime}}\left(4 \xi_{p-k}^{f}\right) B_{n}\left(4 \xi_{p}^{f}\right) .
\end{aligned}
$$

We can slightly simplify the second line after the equality in the above equation using the on-shell condition as

$$
2\left[\left(p_{\|}-k_{\|}\right) \cdot p_{\|}-m_{f}^{2}\right]=p_{\|}^{2}-m_{f}^{2}+\left(p_{\|}-k_{\|}\right)^{2}-m_{f}^{2}-k_{\|}^{2}=2\left|q_{f} B\right|\left(n+n^{\prime}-\xi_{k}^{f}\right),
$$

where we used $k_{\|}^{2}=\boldsymbol{k}_{\perp}^{2}=2\left|q_{f} B\right| \xi_{k}^{f}$ (i.e., onshell-ness of massless gluons). To perform the integration of eq. (4.53) with respect to the transverse momentum, we need formulas of 
(generalized) Laguerre polynomials:

$$
\begin{aligned}
\alpha_{n, n^{\prime}}\left(\xi_{k}^{f}\right) & :=2\left|q_{f} B\right|(-1)^{n-n^{\prime}} \int \frac{\mathrm{d}^{2} p_{\perp}}{(2 \pi)^{2}} \mathrm{e}^{-2 \xi_{p}^{f}} L_{n}\left(4 \xi_{p}^{f}\right) \mathrm{e}^{-2 \xi_{p-k}^{f}} L_{n^{\prime}}\left(4 \xi_{p-k}^{f}\right) \\
& =\frac{\left|q_{f} B\right|}{2 \pi} \mathrm{e}^{-\xi_{k}^{f}} \frac{n !}{n^{\prime} !}\left(\xi_{k}^{f}\right)^{n^{\prime}-n}\left[L_{n}^{\left(n^{\prime}-n\right)}\left(\xi_{k}^{f}\right)\right]^{2}, \\
\beta_{n, n^{\prime}}\left(\xi_{k}^{f}\right) & :=2\left|q_{f} B\right|(-1)^{n-n^{\prime}} \int \frac{\mathrm{d}^{2} p_{\perp}}{(2 \pi)^{2}} \mathrm{e}^{-2 \xi_{p}^{f}} L_{n-1}^{(1)}\left(4 \xi_{p}^{f}\right) \mathrm{e}^{-2 \xi_{p-k}^{f}} L_{n^{\prime}-1}^{(1)}\left(4 \xi_{p-k}^{f}\right) \boldsymbol{p}_{\perp} \cdot\left(\boldsymbol{p}_{\perp}-\boldsymbol{k}_{\perp}\right) \\
& =\frac{\left|q_{f} B\right|}{2 \pi} \mathrm{e}^{-\xi_{k}^{f}} \frac{n !}{n^{\prime} !}\left(\xi_{k}^{f}\right)^{n^{\prime}-n} n^{\prime} L_{n-1}^{\left(n^{\prime}-n\right)}\left(\xi_{k}^{f}\right) L_{n}^{\left(n^{\prime}-n\right)}\left(\xi_{k}^{f}\right) .
\end{aligned}
$$

With these formulas and the explicit forms of eqs. (3.7), (3.8), and (3.9), we find,

$$
\begin{aligned}
& \int \frac{\mathrm{d}^{2} p_{\perp}}{(2 \pi)^{2}} \operatorname{tr}\left[\gamma_{\mu} S_{n}^{f}(p) \gamma^{\mu} S_{n^{\prime}}^{f}(p-k)\right] \\
& =4 m_{f}^{2}\left(\alpha_{n, n^{\prime}}+\alpha_{n-1, n^{\prime}-1}\right)-4\left|q_{f} B\right|\left(n+n^{\prime}-\xi_{k}^{f}\right)\left(\alpha_{n-1, n^{\prime}}+\alpha_{n, n^{\prime}-1}\right)+16\left|q_{f} B\right| \beta_{n, n^{\prime}} \\
& =\frac{\left|q_{f} B\right|}{2 \pi} \mathrm{e}^{-\xi_{k}^{f}} \frac{n !}{n^{\prime} !}\left(\xi_{k}^{f}\right)^{n^{\prime}-n}\left[4 m_{f}^{2} F\left(n, n^{\prime}, \xi_{k}^{f}\right)-4\left|q_{f} B\right|\left(n+n^{\prime}-\xi_{k}^{f}\right) \frac{1}{\xi_{k}^{f}}\left\{\frac{\left(\xi_{k}^{f}\right)^{2}}{n}\left[L_{n-1}^{\left(n^{\prime}-n+1\right)}\left(\xi_{k}^{f}\right)\right]^{2}\right.\right. \\
& \left.\left.\quad+n^{\prime}\left[L_{n}^{\left(n^{\prime}-n-1\right)}\left(\xi_{k}^{f}\right)\right]^{2}\right\}+16\left|q_{f} B\right| n^{\prime} L_{n-1}^{\left(n^{\prime}-n\right)}\left(\xi_{k}^{f}\right) L_{n}^{\left(n^{\prime}-n\right)}\left(\xi_{k}^{f}\right)\right] .
\end{aligned}
$$

Using the following relation,

$$
\frac{\xi^{2}}{n}\left[L_{n-1}^{\left(n^{\prime}-n+1\right)}(\xi)\right]^{2}+n^{\prime}\left[L_{n}^{\left(n^{\prime}-n-1\right)}(\xi)\right]^{2}=\left(n^{\prime}+n\right) F\left(n, n^{\prime}, \xi\right)-4 n^{\prime} L_{n}^{\left(n^{\prime}-n\right)}(\xi) L_{n-1}^{\left(n^{\prime}-n\right)}(\xi),
$$

we eventually obtain $X\left(n, n^{\prime}, \xi\right)$ in a form as given in eq. (4.50).

\subsection{Matrix elements}

We should solve $\chi$ to obtain the longitudinal conductivity from eq. (4.21), and for this, we should invert the collision operator $\mathcal{L}$. In actual numerical calculations we need to represent the operator in a matrix form with appropriate bases. Once we prepare complete sets of $\eta$ and $\chi$, using the inner product we defined in eq. (4.17), we can express $(\eta, \mathcal{L} \chi)$ in the language of the matrix algebra as

$$
\begin{aligned}
(\eta, \mathcal{L} \chi)= & \sum_{n_{1}, n_{2}, l_{1}, l_{2}, f_{1}, f_{2}}\left(c_{f_{1} n_{1} l_{1}}^{\eta} \mathcal{L}_{f_{1} n_{1} l_{1} ; f_{2} n_{2} l_{2}}^{q q} c_{f_{2} n_{2} l_{2}}^{\chi}+c_{f_{1} n_{1} l_{1}}^{\eta} \mathcal{L}_{f_{1} n_{1} l_{1} ; f_{2} n_{2} l_{2}}^{q \bar{q}} \bar{c}_{f_{2} n_{2} l_{2}}^{\chi}\right. \\
& \left.+\bar{c}_{f_{1} n_{1} l_{1}}^{\eta} \mathcal{L}_{f_{1} n_{1} l_{1} ; f_{2} n_{2} l_{2}}^{\bar{q} q} c_{f_{2} n_{2} l_{2}}^{\chi}+\bar{c}_{f_{1} n_{1} l_{1}}^{\eta} \mathcal{L}_{f_{1} n_{1} l_{1} ; f_{2} n_{2} l_{2}}^{\bar{q} \bar{c}} \bar{c}_{f_{2} n_{2} l_{2}}^{\chi}\right) \\
& +\sum_{n, m, l_{1}, l_{2}, f}\left(\bar{c}_{f n l_{1}}^{\eta} \mathcal{L}_{f n l_{1} ; m l_{2}}^{\bar{q} g} \tilde{c}_{m l_{2}}^{\chi}+\tilde{c}_{m l_{1}}^{\eta} \mathcal{L}_{m l_{1} ; f n l_{2}}^{g q} c_{f n l_{2}}^{\chi}\right. \\
& \left.+\tilde{c}_{m l_{1}}^{\eta} \mathcal{L}_{m l_{1} ; f n l_{2}}^{\bar{q}} \bar{c}_{f n l_{2}}^{\chi}+c_{f n l_{1}}^{\eta} \mathcal{L}_{f n l_{1} ; m l_{2}}^{q g} \tilde{c}_{m l_{2}}^{\chi}\right) \\
& \left.+\sum_{m_{1}, m_{2}, l_{1}, l_{2}} \tilde{c}_{m_{1} l_{1}}^{\eta} \mathcal{L}_{m_{1} l_{1} ; m_{2} l_{2}}^{g g} \tilde{c}_{m_{2} l_{2}}^{\chi}\right]
\end{aligned}
$$


where $\left(c_{f n l}^{\eta}, \bar{c}_{f n l}^{\eta}, \tilde{c}_{m l}^{\eta}\right)$ and $\left(c_{f n l}^{\chi}, \bar{c}_{f n l}^{\chi}, \tilde{c}_{m l}^{\chi}\right)$ represent the components of $\eta=\left(\eta_{p}, \bar{\eta}_{p}, \tilde{\eta}_{k}\right)^{T}$ and $\chi=\left(\chi_{p}, \bar{\chi}_{p}, \tilde{\eta}_{k}\right)^{T}$, respectively, with chosen bases. The simplest choice of the bases would be the polynomial one. These vectors are then expanded as

$$
\chi=\left(\begin{array}{c}
\sum_{f, n, l} c_{f n l}^{\chi} \delta^{n f} d_{l}\left(p_{z}\right) \\
\sum_{f, n, l} \bar{c}_{f n l}^{\chi} \delta^{n f} d_{l}\left(p_{z}\right) \\
\sum_{m, l} \tilde{c}_{m l}^{\chi} d_{l}\left(k_{z}\right) b_{m}\left(k_{\perp}\right)
\end{array}\right), \quad \eta=\left(\begin{array}{c}
\sum_{f, n, l} c_{f n l}^{\eta} \delta^{n f} d_{l}\left(p_{z}\right) \\
\sum_{f, n, l} \bar{c}_{f n l}^{\eta} \delta^{n f} d_{l}\left(p_{z}\right) \\
\sum_{m, l} \tilde{c}_{m l}^{\eta} d_{l}\left(k_{z}\right) b_{m}\left(k_{\perp}\right)
\end{array}\right),
$$

where the $m$-th component of $\delta^{n f}$ is $\delta_{m}^{n \times N_{f}+f}$ with the flavor number $N_{f}$, and $d_{l}\left(p_{z}\right)$ and $b_{m}\left(k_{\perp}\right)$ are polynomial bases, namely, $d_{l}\left(p_{z}\right)=\hat{p}_{z}\left|p_{z}\right|^{l}$ and $b_{m}\left(k_{\perp}\right)=k_{\perp}^{m}$. Now, using these basis functions, we can explicitly write down the matrix elements of the operator $\mathcal{L}$. The diagonal parts (i.e., $q q, \bar{q} \bar{q}$, and $g g$ components) are

$$
\begin{aligned}
& \mathcal{L}_{f_{1} n_{1} l_{1} ; f_{2} n_{2} l_{2}}^{q q}=\delta_{f_{1} f_{2}}\left\{-\frac{\delta_{n_{1} n_{2}}}{2} \sum_{n^{\prime}=0}^{n_{1}-1} \int \frac{\mathrm{d} p_{z}}{2 \pi} \frac{1}{2 \varepsilon_{f_{1} n_{1}}} \int^{\prime} \frac{\mathrm{d} p_{z}^{\prime}}{2 \pi} \frac{1}{2 \varepsilon_{f_{1} n^{\prime}}} X\left(n_{1}, n^{\prime}, \xi_{-}\right) f_{\mathrm{eq}}(p)\left[1-f_{\mathrm{eq}}\left(p^{\prime}\right)\right]\right. \\
& \times\left[1+g_{\text {eq }}(k)\right] d_{l_{1}}\left(p_{z}\right) d_{l_{2}}\left(p_{z}\right)-\frac{\delta_{n_{1} n_{2}}}{2} \sum_{n=n_{2}+1}^{\infty} \int \frac{\mathrm{d} p_{z}}{2 \pi} \frac{1}{2 \varepsilon_{f_{1} n}} \int^{\prime} \frac{\mathrm{d} p_{z}^{\prime}}{2 \pi} \frac{1}{2 \varepsilon_{f_{1} n_{2}}} X\left(n, n_{2}, \xi_{-}\right) f_{\text {eq }}(p) \\
& \times\left[1-f_{\mathrm{eq}}\left(p^{\prime}\right)\right]\left[1+g_{\mathrm{eq}}(k)\right] d_{l_{1}}\left(p_{z}^{\prime}\right) d_{l_{2}}\left(p_{z}^{\prime}\right)+\frac{\delta_{n_{1} n_{2}}}{2} \sum_{n^{\prime}=0}^{\infty} \int \frac{\mathrm{d} p_{z}}{2 \pi} \frac{1}{2 \varepsilon_{f_{1} n_{1}}} \int \frac{\mathrm{d} p_{z}^{\prime}}{2 \pi} \frac{1}{2 \varepsilon_{f_{1} n^{\prime}}} X\left(n_{1}, n^{\prime}, \xi_{+}\right) \\
& \times f_{\text {eq }}(p) \bar{f}_{\text {eq }}\left(p^{\prime}\right)\left[1+g_{\text {eq }}(k)\right] d_{l_{1}}\left(p_{z}\right) d_{l_{2}}\left(p_{z}\right)+\frac{\vartheta\left(n_{1}-n_{2}-1\right)}{2} \int \frac{\mathrm{d} p_{z}}{2 \pi} \frac{1}{2 \varepsilon_{f_{1} n_{1}}} \int^{\prime} \frac{\mathrm{d} p_{z}^{\prime}}{2 \pi} \frac{1}{2 \varepsilon_{f_{1} n_{2}}} \\
& \times X\left(n_{1}, n_{2}, \xi_{-}\right) f_{\mathrm{eq}}(p)\left[1-f_{\mathrm{eq}}\left(p^{\prime}\right)\right]\left[1+g_{\mathrm{eq}}(k)\right] d_{l_{1}}\left(p_{z}\right) d_{l_{2}}\left(p_{z}^{\prime}\right)+\frac{\vartheta\left(n_{2}-n_{1}-1\right)}{2} \int \frac{\mathrm{d} p_{z}}{2 \pi} \frac{1}{2 \varepsilon_{f_{1} n_{2}}} \\
& \left.\times \int^{\prime} \frac{\mathrm{d} p_{z}^{\prime}}{2 \pi} \frac{1}{2 \varepsilon_{f_{1} n_{1}}} X\left(n_{2}, n_{1}, \xi_{-}\right) f_{\mathrm{eq}}(p)\left[1-f_{\mathrm{eq}}\left(p^{\prime}\right)\right]\left[1+g_{\mathrm{eq}}(k)\right] d_{l_{1}}\left(p_{z}^{\prime}\right) d_{l_{2}}\left(p_{z}\right)\right\} \\
& \mathcal{L}_{f_{1} n_{1} l_{1} ; f_{2} n_{2} l_{2}}^{\bar{q} \bar{q}}=\delta_{f_{1} f_{2}}\left\{-\frac{\delta_{n_{1} n_{2}}}{2} \sum_{n^{\prime}=0}^{n_{1}-1} \int \frac{\mathrm{d} p_{z}}{2 \pi} \frac{1}{2 \varepsilon_{f_{1} n_{1}}} \int^{\prime} \frac{\mathrm{d} p_{z}^{\prime}}{2 \pi} \frac{1}{2 \varepsilon_{f_{1} n^{\prime}}} X\left(n_{1}, n^{\prime}, \xi_{-}\right) \bar{f}_{\mathrm{eq}}(p)\left[1-\bar{f}_{\mathrm{eq}}\left(p^{\prime}\right)\right]\right. \\
& \times\left[1+g_{\mathrm{eq}}(k)\right] d_{l_{1}}\left(p_{z}\right) d_{l_{2}}\left(p_{z}\right)-\frac{\delta_{n_{1} n_{2}}}{2} \sum_{n=n_{2}+1}^{\infty} \int \frac{\mathrm{d} p_{z}}{2 \pi} \frac{1}{2 \varepsilon_{f_{1} n}} \int^{\prime} \frac{\mathrm{d} p_{z}^{\prime}}{2 \pi} \frac{1}{2 \varepsilon_{f_{1} n_{2}}} X\left(n, n_{2}, \xi_{-}\right) \bar{f}_{\mathrm{eq}}(p) \\
& \times\left[1-\bar{f}_{\mathrm{eq}}\left(p^{\prime}\right)\right]\left[1+g_{\mathrm{eq}}(k)\right] d_{l_{1}}\left(p_{z}^{\prime}\right) d_{l_{2}}\left(p_{z}^{\prime}\right)+\frac{\delta_{n_{1} n_{2}}}{2} \sum_{n=0}^{\infty} \int \frac{\mathrm{d} p_{z}}{2 \pi} \frac{1}{2 \varepsilon_{f_{1} n}} \int \frac{\mathrm{d} p_{z}^{\prime}}{2 \pi} \frac{1}{2 \varepsilon_{f_{1} n_{2}}} X\left(n, n_{2}, \xi_{+}\right) \\
& \times f_{\mathrm{eq}}(p) \bar{f}_{\mathrm{eq}}\left(p^{\prime}\right)\left[1+g_{\mathrm{eq}}(k)\right] d_{l_{1}}\left(p_{z}^{\prime}\right) d_{l_{2}}\left(p_{z}^{\prime}\right)+\frac{\vartheta\left(n_{1}-n_{2}-1\right)}{2} \int \frac{\mathrm{d} p_{z}}{2 \pi} \frac{1}{2 \varepsilon_{f_{1} n_{1}}} \int^{\prime} \frac{\mathrm{d} p_{z}^{\prime}}{2 \pi} \frac{1}{2 \varepsilon_{f_{1} n_{2}}} \\
& \times X\left(n_{1}, n_{2}, \xi_{-}\right) \bar{f}_{\mathrm{eq}}(p)\left[1-\bar{f}_{\mathrm{eq}}\left(p^{\prime}\right)\right]\left[1+g_{\mathrm{eq}}(k)\right] d_{l_{1}}\left(p_{z}\right) d_{l_{2}}\left(p_{z}^{\prime}\right)+\frac{\vartheta\left(n_{2}-n_{1}-1\right)}{2} \int \frac{\mathrm{d} p_{z}}{2 \pi} \frac{1}{2 \varepsilon_{f_{1} n_{2}}} \\
& \left.\times \int^{\prime} \frac{\mathrm{d} p_{z}^{\prime}}{2 \pi} \frac{1}{2 \varepsilon_{f_{1} n_{1}}} X\left(n_{2}, n_{1}, \xi_{-}\right) \bar{f}_{\mathrm{eq}}(p)\left[1-\bar{f}_{\mathrm{eq}}\left(p^{\prime}\right)\right]\left[1+g_{\mathrm{eq}}(k)\right] d_{l_{1}}\left(p_{z}^{\prime}\right) d_{l_{2}}\left(p_{z}\right)\right\},
\end{aligned}
$$


and

$$
\begin{aligned}
& \mathcal{L}_{m_{1} l_{1} ; m_{2} l_{2}}^{g g}=\frac{1}{2} \sum_{f}\left\{-\sum_{n=1}^{\infty} \sum_{n^{\prime}=0}^{n-1} \int \frac{\mathrm{d} p_{z}}{2 \pi} \frac{1}{2 \varepsilon_{f n}} \int^{\prime} \frac{\mathrm{d} p_{z}^{\prime}}{2 \pi} \frac{1}{2 \varepsilon_{f n^{\prime}}} X\left(n, n^{\prime}, \xi_{-}\right) f_{\mathrm{eq}}(p)\left[1-f_{\mathrm{eq}}\left(p^{\prime}\right)\right]\right. \\
& \times\left[1+g_{\mathrm{eq}}(k)\right] d_{l_{1}}\left(k_{z}\right) d_{l_{2}}\left(k_{z}\right) b_{m_{1}}\left(k_{\perp}\right) b_{m_{2}}\left(k_{\perp}\right)-\sum_{n=1}^{\infty} \sum_{n^{\prime}=0}^{n-1} \int \frac{\mathrm{d} p_{z}}{2 \pi} \frac{1}{2 \varepsilon_{f n}} \int \frac{\mathrm{d} p_{z}^{\prime}}{2 \pi} \frac{1}{2 \varepsilon_{f n^{\prime}}} \\
& \times X\left(n, n^{\prime}, \xi_{-}\right) \bar{f}_{\mathrm{eq}}(p)\left[1-\bar{f}_{\mathrm{eq}}\left(p^{\prime}\right)\right]\left[1+g_{\mathrm{eq}}(k)\right] d_{l_{1}}\left(k_{z}\right) d_{l_{2}}\left(k_{z}\right) b_{m_{1}}\left(k_{\perp}\right) b_{m_{2}}\left(k_{\perp}\right) \\
& +\sum_{n=0}^{\infty} \sum_{n^{\prime}=0}^{\infty} \int \frac{\mathrm{d} p_{z}}{2 \pi} \frac{1}{2 \varepsilon_{f n}} \int \frac{\mathrm{d} p_{z}^{\prime}}{2 \pi} \frac{1}{2 \varepsilon_{f n^{\prime}}} X\left(n, n^{\prime}, \xi_{+}\right) f_{\mathrm{eq}}(p) \bar{f}_{\mathrm{eq}}\left(p^{\prime}\right)\left[1+g_{\mathrm{eq}}(k)\right] \\
& \left.\times d_{l_{1}}\left(k_{z}\right) d_{l_{2}}\left(k_{z}\right) b_{m_{1}}\left(k_{\perp}\right) b_{m_{2}}\left(k_{\perp}\right)\right\} .
\end{aligned}
$$

In the same way the off-diagonal parts are

$$
\begin{aligned}
& \mathcal{L}_{f_{1} n_{1} l_{1} ; f_{2} n_{2} l_{2}}^{q \bar{q}}= \frac{\delta_{f_{1} f_{2}}}{2} \int \frac{\mathrm{d} p_{z}}{2 \pi} \frac{1}{2 \varepsilon_{f_{1} n_{1}}} \int \frac{\mathrm{d} p_{z}^{\prime}}{2 \pi} \frac{1}{2 \varepsilon_{f_{1} n_{2}}} \\
& \times X\left(n_{1}, n_{2}, \xi_{+}\right) f_{\mathrm{eq}}(p) \bar{f}_{\mathrm{eq}}\left(p^{\prime}\right)\left[1+g_{\mathrm{eq}}(k)\right] d_{l_{1}}\left(p_{z}\right) d_{l_{2}}\left(p_{z}^{\prime}\right), \\
& \mathcal{L}_{f n_{1} l_{1} ; m l_{2}}^{q g}=\frac{1}{2} \sum_{n=n_{1}+1}^{\infty} \int \frac{\mathrm{d} p_{z}}{2 \pi} \frac{1}{2 \varepsilon_{f n}} \int \frac{\mathrm{d} p_{z}^{\prime}}{2 \pi} \frac{-1}{2 \varepsilon_{f n_{1}}} X\left(n, n_{1}, \xi_{-}\right) f_{\mathrm{eq}}(p)\left[1-f_{\mathrm{eq}}\left(p^{\prime}\right)\right]\left[1+g_{\mathrm{eq}}(k)\right] \\
& \times d_{l_{1}}\left(p_{z}^{\prime}\right) d_{l_{2}}\left(k_{z}\right) b_{m}\left(k_{\perp}\right)-\frac{1}{2} \sum_{n^{\prime}=0}^{n_{1}-1} \int \frac{\mathrm{d} p_{z}}{2 \pi} \frac{1}{2 \varepsilon_{f n_{1}}} \int \frac{\mathrm{d} p_{z}^{\prime}}{2 \pi} \frac{-1}{2 \varepsilon_{f n^{\prime}}} X\left(n_{1}, n^{\prime}, \xi_{-}\right) f_{\mathrm{eq}}(p)\left[1-f_{\mathrm{eq}}\left(p^{\prime}\right)\right] \\
& \times\left[1+g_{\mathrm{eq}}(k)\right] d_{l_{1}}\left(p_{z}\right) d_{l_{2}}\left(k_{z}\right) b_{m}\left(k_{\perp}\right)-\frac{1}{2} \sum_{n^{\prime}=0}^{\infty} \int \frac{\mathrm{d} p_{z}}{2 \pi} \frac{1}{2 \varepsilon_{f n_{1}}} \int \frac{\mathrm{d} p_{z}^{\prime}}{2 \pi} \frac{1}{2 \varepsilon_{f n^{\prime}}} X\left(n_{1}, n^{\prime}, \xi_{+}\right) \\
& \quad \times f_{\mathrm{eq}}(p) \bar{f}_{\mathrm{eq}}\left(p^{\prime}\right)\left[1+g_{\mathrm{eq}}(k)\right] d_{l_{1}}\left(p_{z}\right) d_{l_{2}}\left(k_{z}\right) b_{m}\left(k_{\perp}\right),
\end{aligned}
$$

and

$$
\begin{aligned}
& \mathcal{L}_{f n_{1} l_{1} ; m l_{2}}^{\bar{q} g}=\frac{1}{2} \sum_{n=n_{1}+1}^{\infty} \int \frac{\mathrm{d} p_{z}}{2 \pi} \frac{1}{2 \varepsilon_{f n}} \int \frac{\mathrm{d} p_{z}^{\prime}}{2 \pi} \frac{-1}{2 \varepsilon_{f n_{1}}} X\left(n, n_{1}, \xi_{-}\right) \bar{f}_{\mathrm{eq}}(p)\left[1-\bar{f}_{\mathrm{eq}}\left(p^{\prime}\right)\right]\left[1+g_{\mathrm{eq}}(k)\right] \\
& \times d_{l_{1}}\left(p_{z}^{\prime}\right) d_{l_{2}}\left(k_{z}\right) b_{m}\left(k_{\perp}\right)-\frac{1}{2} \sum_{n^{\prime}=0}^{n_{1}-1} \int \frac{\mathrm{d} p_{z}}{2 \pi} \frac{1}{2 \varepsilon_{f n_{1}}} \int \frac{\mathrm{d} p_{z}^{\prime}}{2 \pi} \frac{-1}{2 \varepsilon_{f n^{\prime}}} X\left(n_{1}, n^{\prime}, \xi_{-}\right) \bar{f}_{\mathrm{eq}}(p)\left[1-\bar{f}_{\mathrm{eq}}\left(p^{\prime}\right)\right] \\
& \times\left[1+g_{\mathrm{eq}}(k)\right] d_{l_{1}}\left(p_{z}\right) d_{l_{2}}\left(k_{z}\right) b_{m}\left(k_{\perp}\right)-\frac{1}{2} \sum_{n=0}^{\infty} \int \frac{\mathrm{d} p_{z}}{2 \pi} \frac{1}{2 \varepsilon_{f n}} \int \frac{\mathrm{d} p_{z}^{\prime}}{2 \pi} \frac{1}{2 \varepsilon_{f n_{1}}} X\left(n, n_{1}, \xi_{+}\right) \\
& \times f_{\mathrm{eq}}(p) \bar{f}_{\mathrm{eq}}\left(p^{\prime}\right)\left[1+g_{\mathrm{eq}}(k)\right] d_{l_{1}}\left(p_{z}^{\prime}\right) d_{l_{2}}\left(k_{z}\right) b_{m}\left(k_{\perp}\right) .
\end{aligned}
$$

Other off-diagonal components are given by the symmetric properties of $\mathcal{L}$, that is,

$$
\mathcal{L}_{f_{1} n_{1} l_{1} ; f_{2} n_{2} l_{2}}^{\bar{q} q}=\mathcal{L}_{f_{2} n_{2} l_{2} ; f_{1} n_{1} l_{1}}^{q \bar{q}}, \quad \mathcal{L}_{n_{1} l_{1} ; f_{2} n_{2} l_{2}}^{g q}=\mathcal{L}_{f_{2} n_{2} l_{2} ; n_{1} l_{1}}^{q g}, \quad \mathcal{L}_{n_{1} l_{1} ; f_{2} n_{2} l_{2}}^{g \bar{q}}=\mathcal{L}_{f_{2} n_{2} l_{2} ; n_{1} l_{1}}^{\bar{q} g} .
$$


Now that we get all the matrix elements, we can numerically take the inverse to obtain $\mathcal{L}^{-1}$, which should be multiplied to $\mathcal{S}$. Then, we also need to know the matrix representation of $\mathcal{S}$ projected with the same chosen bases:

$$
\begin{aligned}
& S_{f n l}=N_{\mathrm{c}} \frac{\left|q_{f} B\right|}{2 \pi} \alpha_{n} \int \frac{\mathrm{d} p_{z}}{2 \pi} f_{\mathrm{eq}}(p)\left[1-f_{\mathrm{eq}}(p)\right]\left(q_{f} \frac{p_{z}}{\varepsilon_{f n}}-p_{z} \frac{n_{e}}{\mathcal{E}+\mathcal{P}_{z}}\right) d_{l}\left(p_{z}\right), \\
& \bar{S}_{f n l}=N_{\mathrm{c}} \frac{\left|q_{f} B\right|}{2 \pi} \alpha_{n} \int \frac{\mathrm{d} p_{z}}{2 \pi} \bar{f}_{\mathrm{eq}}(p)\left[1-\bar{f}_{\mathrm{eq}}(p)\right]\left(-q_{f} \frac{p_{z}}{\varepsilon_{f n}}-p_{z} \frac{n_{e}}{\mathcal{E}+\mathcal{P}_{z}}\right) d_{l}\left(p_{z}\right), \\
& \tilde{S}_{m l}=2\left(N_{\mathrm{c}}^{2}-1\right) \int \frac{\mathrm{d}^{3} k}{(2 \pi)^{3}} g_{\mathrm{eq}}(k)\left[1+g_{\mathrm{eq}}(k)\right]\left(-k_{z} \frac{n_{e}}{\mathcal{E}+\mathcal{P}_{z}}\right) d_{l}\left(k_{z}\right) b_{m}\left(k_{\perp}\right) .
\end{aligned}
$$

Using these expressions we can calculate $\chi=\mathcal{Q} \mathcal{L}^{-1} \mathcal{Q S}$, namely, $\left(c_{f n l}^{\chi}, \bar{c}_{f n l}^{\chi}, \tilde{c}_{m l}^{\chi}\right)$ numerically, and we reexpress $\sigma_{\|}=\beta(\mathcal{S}, \chi)$ in eq. (4.21) with these components, which leads to

$$
\sigma_{\|}=\beta \sum_{f, n, l}\left(c_{f n l}^{\chi} S_{f n l}+\bar{c}_{f n l}^{\chi} \bar{S}_{f n l}\right)+\beta \sum_{m, l} \tilde{c}_{m l}^{\chi} \tilde{S}_{m l}
$$

This is our final expression for $\sigma_{\|}$from the analytical side, and we must now switch gear to numerical computations. Before proceeding to the numerical results, we would point out a crucial difference between zero and finite densities. For $\mu_{f}=0$, the solution satisfies $c_{f n l}^{\chi}=$ $-\bar{c}_{f n l}^{\chi}$, and $\tilde{c}_{m l}^{\chi}=0$ due to charge conjugation symmetry. In this case, the conductivity of the whole system can be simply a sum of contributions from different flavor sectors and there appears no mixing term. In contrast, for $\mu_{f} \neq 0$, different flavors mix together through the gluonic matrix elements, $\mathcal{L}^{q g}$ and $\mathcal{L}^{\bar{q} g}$.

\subsection{Numerical results and approximation checks}

We have spelled out all necessary formulas explicitly, and we will present numerical results from now on. In principle the Landau level sum goes to infinity, but for numerical calculations we should truncate it at a certain maximum value of $n$, which we denote $n_{\text {max }}$. In this work we check the convergence up to $n_{\max }=6$ at zero density, and for finite density we take $n_{\max }=4$, for the computation is much demanding at finite density. Also, in practice, we should truncate the sum in the angular momentum $l$, for which we choose $l_{\max }=2$. We have numerically confirmed that this $l$ sum has fast convergence and $l_{\max }=2$ is a very good approximation. For all the numerical results presented below, we fix the QCD coupling constant as $g^{2} /(4 \pi)=0.3$, the system temperature as $T=200 \mathrm{MeV}$ which is well above the QCD phase transition.

Figure 5 shows our numerical results for $\sigma_{\|} / T$ as a function of the current quark mass, $m_{q}$, for a single flavor case. For convenience we set the electric charge carried by this flavor as $q=e$. The magnetic field is chosen to be $e B=10 m_{\pi}^{2}$ and the density is vanishing with $\mu=0$ for the moment. We will discuss the density dependence later.

It is evident from figure 5 that the LLLA has rapid enhancement as $m_{q}$ approaches zero. In this way we can clearly understand that the LLLA breaks down for $\sigma_{\|}\left(m_{q} \rightarrow 0\right)$ even though the magnetic field, $e B=10 m_{\pi}^{2}$, is stronger than typical QCD energy scales $\sim m_{\pi}$. The singular behavior is, as we explained in the introduction, attributed to the 


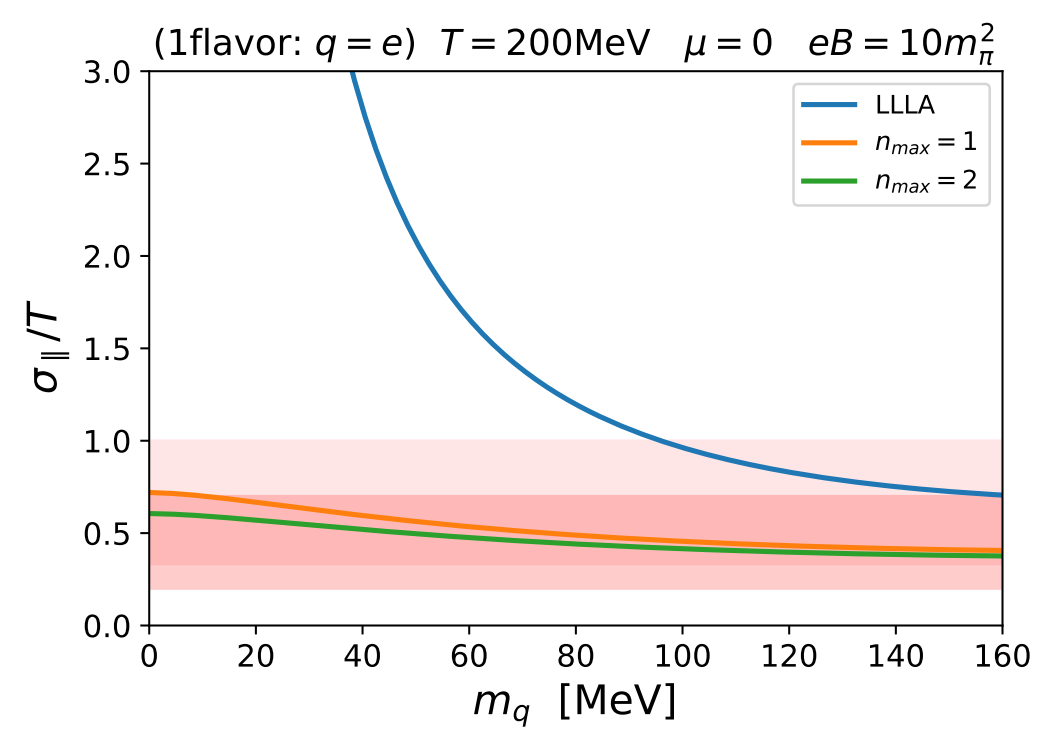

Figure 5. Mass dependence of the longitudinal electric conductivity for a single flavor case with $q_{f}=e$. In the small mass region the LLLA blows up, while the results at $n_{\max }=1,2$ have regular behavior at $m_{q} \sim 0$. The red shaded bands are the lattice-QCD estimates. See the text for discussions.

phase space restriction. In the LLLA the longitudinal and the transverse dynamics of fermions are decoupled, and the longitudinal scattering in $(1+1)$ dimensions is prohibited for massless fermions due to the energy-momentum conservation. In this sense, only the LLLA is exceptional, and the convergence of the Landau level sum is pretty fast if one goes beyond the LLLA, as seen in figure 5 ; the $n_{\max }=1$ results already give a good approximation close to the $n_{\max }=2$ results. Here, we make one important remark; one might think that $\sigma_{\|}\left(m_{q} \rightarrow 0\right)$ should diverge even beyond the LLLA because, according to the axial Ward identity, the chirality in the massless limit is linearly increasing with time regardless the scattering (except for the sphaleron transition which is suppressed and negligible at weak coupling). This argument is mathematically correct, but physically the divergence signifies a hydrodynamic mode (in a particular hydrodynamic regime; see discussions in Introduction). In fact, as we closely explained, the conserved quantities such as the energy momentum tensor and the electric charge constitute the hydrodynamic modes which should be subtracted; otherwise, they lead to divergence. In the small mass limit the axial charge is approximately conserved, thus it forms another hydrodynamic mode. We did not explicitly subtract this additional hydrodynamic mode, but our calculation procedures without coupling to the axial charge automatically drops out such a hydrodynamic mode. More specifically, out treatment of the distributions did not allow for the axial charge beyond the linear response regime, so that the hydrodynamic mode corresponding to the axial charge is excluded.

In figure 5 the red shaded bands represent the lattice-QCD estimates. It is interesting that our results are quantitatively consistent with the lattice-QCD estimates, i.e., the light red region of $1 / 3 \leq \sigma / T \leq 1$ at $T=1.45 T_{\mathrm{c}}$ (for the quark charge squared sum $C_{\mathrm{em}}=1$ 


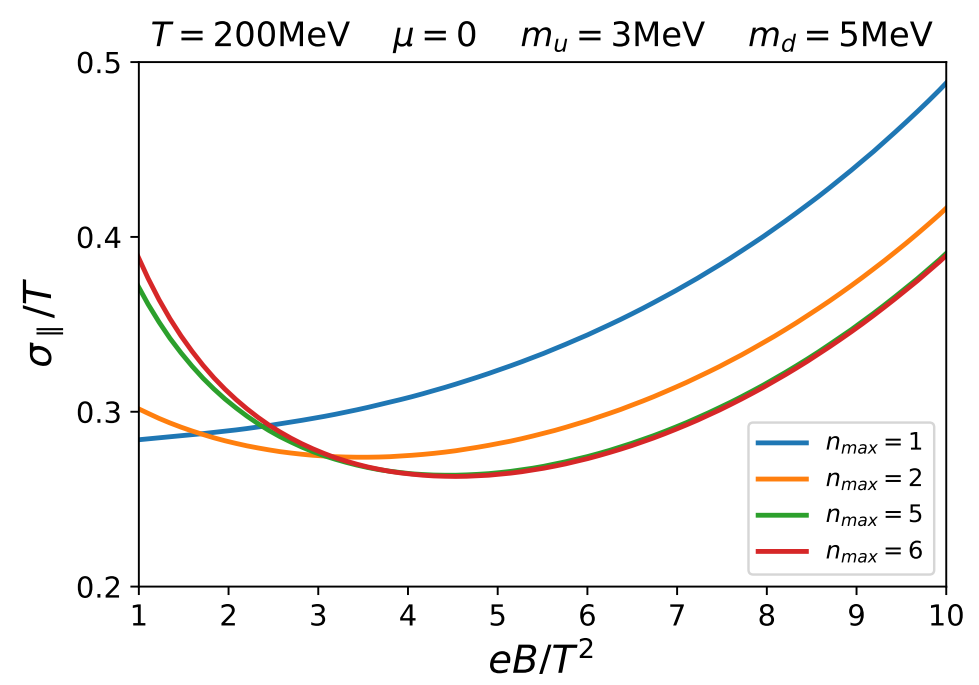

Figure 6. Magnetic dependence of the longitudinal electric conductivity for two favor case for various $n_{\max }$.

which corresponds to our single flavor with $q=e$ ) in ref. [42] and the darker red region of $0.2 \leq \sigma / T \leq 1$ at $T=1.1 T_{\mathrm{c}}$ in ref. [39]. We note that $T_{\mathrm{c}}$ is the critical temperature in quenched QCD, which is substantially larger than the QCD (pseudo) critical temperature. It is thus difficult to translate the lattice temperature, and if we plug the physical QCD critical temperature into $T_{\mathrm{c}}$, our choice of $T=200 \mathrm{MeV}$ is almost comparable to the lattice temperature. As we see next, the $B$ dependence of $\sigma_{\|} / T$ is moderate, at most $30 \%$ changes up to $e B \sim 10 m_{\pi}^{2}$, not order of magnitude differences. Therefore, it would be sensible to make such a comparison of our results at $e B=10 m_{\pi}^{2}$ and the lattice-QCD results at $B=0$. Still, we should emphasize that this is not an apple-to-apple comparison, and we should not overstate the agreement beyond the qualitative level. Nevertheless, the quantitative agreement in figure 5 implies for $B=0$ that $\sigma_{\|} / T<1$ as reported in refs. [41, 42] would be favored and a former large value in ref. [40] is unlikely.

Let us turn to the $e B$ dependence of $\sigma_{\|} / T$, which is plotted in figure 6 . In this case we include $u$ and $d$ flavors with physical quark masses $\left(m_{u}=3 \mathrm{MeV}\right.$ and $\left.m_{d}=5 \mathrm{MeV}\right)$ and physical electric charges $\left(q_{u}=\frac{2}{3} e\right.$ and $\left.q_{d}=-\frac{1}{3} e\right)$. If $e B$ goes smaller, the convergence of the Landau level sum would becomes slower, and in fact, figure 6 shows that the $n_{\max }=1$ and the $n_{\max }=2$ results behave quite differently as $e B \rightarrow 0$. Still, we can observe a tendency of fast convergence from the $n_{\max }=5$ and the $n_{\max }=6$ results for any magnetic strength. Figure 6 is our main result, and to emphasize its nature as realization of the negative magnetoresistance, the resistance, $\rho_{\|}=1 / \sigma_{\|}$was presented in figure 1 as displayed in the very beginning of this paper.

It is a very interesting question how such a nonmonotonic shape emerges; actually the experimental data also shows a dip at small magnetic field, which qualitatively agrees with our results. In our calculation the nonmonotonicy appears as a result of competing two effects. For large $e B$ the LLL contribution will become dominant, as we closely check in the next subsection, and then $\sigma_{\|}$is linearly proportional to $e B$, which can be intuitively 


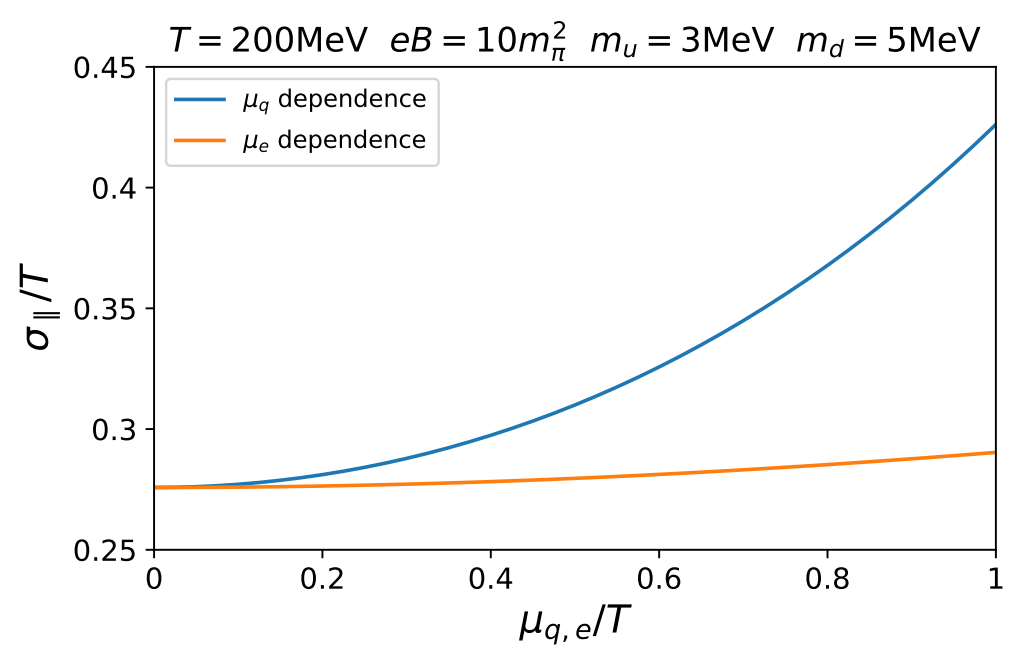

Figure 7. Chemical potential dependence of the longitudinal electric conductivity for two flavor case. The upper line is for the quark chemical potential, while the lower one is for the electric chemical potential coupled to the electric charge.

understood from the fact that the charge carrier increases then. For small $e B$, on the other hand, contributions from higher Landau levels lead to a larger interaction cross section due to the phase space factor, which lowers $\sigma_{\|}$with increasing $e B$. Interestingly, in the intermediate region of $e B$, the behavior of $\sigma_{\|}$looks quadratic, as perceived from figure 6 . In this way our results capture all qualitative features of the experimental data, while quantitative details would depend on underlying theory.

Finally in this subsection, we shall consider the dependence of quark chemical potential as shown in figure 7 . We can notice that the dependence of the quark chemical potential $\mu_{q}$ is mild, and that of the electric chemical potential $\mu_{e}$ is even more suppressed.

\subsection{Asymptotics at strong magnetic field toward the LLLA}

It would be an instructive check to confirm that the known results in the LLLA are correctly recovered from eq. (4.71) in the limit of $q_{f} B \gg T^{2}$ (and $\mu_{f}=0$ for simplicity). Under this limit only the LLL contributes to physical observables. Since the synchrotron radiation requires changes with respect to the Landau levels, we can safely discard the radiation terms and the absorption terms. For the pair annihilation process, $X\left(n=0, n^{\prime}=0, \xi\right)$ in eq. (4.50) simplifies as

$$
X\left(0,0, \xi_{+}^{f}\right)=4 m_{f}^{2} g^{2} N_{\mathrm{c}} C_{F} \frac{\left|q_{f} B\right|}{2 \pi} \mathrm{e}^{-\xi_{+}^{0}}
$$

with $\xi_{+}^{0}=\left[\left(\sqrt{p_{z}^{2}+m_{f}^{2}}+\sqrt{p_{z}^{\prime 2}+m_{f}^{2}}\right)^{2}-\left(p_{z}+p_{z}^{\prime}\right)^{2}\right] /\left(2\left|q_{f} B\right|\right)$ which is nothing but $\xi_{+}$ obtained from eq. (4.44) with $n=n^{\prime}=0$. When $\left|q_{f} B\right|$ is much larger than any other scales, we can approximate $\mathrm{e}^{-\xi_{+}^{0}} \approx 1$ neglecting the dependence on $\xi_{+}^{0}$ which is suppressed 
by $\left|q_{f} B\right|$. Then, the linearized Boltzmann equations reduce to a simple form:

$$
\begin{aligned}
& q_{f} N_{\mathrm{c}} \frac{\left|q_{f} B\right|}{2 \pi} \beta f_{\mathrm{eq}}(p)\left[1-f_{\mathrm{eq}}(p)\right] \frac{p_{z}}{\sqrt{p_{z}^{2}+m_{f}^{2}}} \\
& =4 m_{f}^{2} g^{2} N_{\mathrm{c}} C_{F} \beta \frac{\left|q_{f} B\right|}{2 \pi} \cdot \frac{1}{2} \cdot \frac{1}{2 \varepsilon_{f 0}} \int \frac{\mathrm{d} p_{z}^{\prime}}{2 \pi} \frac{1}{2 \varepsilon_{f 0}^{\prime}} f_{\mathrm{eq}}(p) \bar{f}_{\mathrm{eq}}\left(p^{\prime}\right)\left[1+g_{\mathrm{eq}}(k)\right] \chi_{p},
\end{aligned}
$$

where $\varepsilon_{f 0}=\sqrt{p_{z}^{2}+m_{f}^{2}}$ and $\varepsilon_{f 0}^{\prime}=\sqrt{p_{z}^{\prime 2}+m_{f}^{2}}$. Here, we do not have to consider mixing terms with $\bar{\chi}_{p^{\prime}}$ because $\bar{\chi}_{p^{\prime}}$ is an odd function in terms of $p^{\prime}$ and the integrand is even, so that the integral vanishes. Therefore, in this special limit, $\mathcal{L}$ is not really a matrix and we do not need to take its matrix inversion. Actually, we can easily solve the above Boltzmann equation to find $\chi_{p}$ as

$$
\chi_{p}=\frac{q_{f}}{g^{2} C_{F} m_{f}^{2}}\left[1-f_{\mathrm{eq}}(p)\right] \frac{p_{z}}{\int \frac{\mathrm{d} p_{z}^{\prime}}{2 \pi} \frac{1}{2 \varepsilon_{f 0}^{\prime}} \bar{f}_{\mathrm{eq}}\left(p^{\prime}\right)\left[1+g_{\mathrm{eq}}(k)\right]} .
$$

Thanks to the charge conjugation symmetry, the solution for the antiparticle is $\bar{\chi}_{p}=-\chi_{p}$. Assembling these expressions, we finally arrive at the longitudinal conductivity in the LLLA, i.e.,

$$
\sigma_{\|}=\sum_{f} \frac{N_{\mathrm{c}} \beta}{g^{2} C_{F} m_{f}^{2}} q_{f}^{2} \frac{\left|q_{f} B\right|}{2 \pi} \int \frac{\mathrm{d} p_{z}}{2 \pi} \frac{p_{z}^{2}}{\sqrt{p_{z}^{2}+m_{f}^{2}}} \frac{f_{\mathrm{eq}}(p)\left[1-f_{\mathrm{eq}}(p)\right]^{2}}{\int \frac{\mathrm{d} p_{z}^{\prime}}{2 \pi} \frac{1}{\varepsilon_{f 0}^{\prime}} \bar{f}_{\mathrm{eq}}\left(p^{\prime}\right)\left[1+g_{\mathrm{eq}}(k)\right]},
$$

which is consistent with the results in ref. [37].

Figure 8 is a numerical comparison between the LLLA results (blue curve) and the full results (orange curve). The latter asymptotically approaches the former, but the convergence is very slow (which quantitatively depends on the fermion masses). For the present parameters the LLLA can be a reasonable approximation only for $e B \gtrsim 200 T^{2}$.

We point out that eq. (4.75) is a quite suggestive expression on the analytical level. Obviously, $\sigma_{\|}$diverges in the limit of $m_{f} \rightarrow 0$, and this singular behavior implies that the electric current also diverges. In fact, in $(1+1)$ dimensions, the chiral anomaly predicts a topological current proportional to time for non-interacting fermions, that is, $\partial_{t} j_{z}=$ (const.) or $j_{z} \propto t[4,14]$. In such a case of linear rising current with time, the electric conductivity defined at $k_{0} \rightarrow 0$ diverges. Therefore, this divergent component from the LLL should be attributed to the chiral anomaly. One might think that $\sigma_{\|} \rightarrow \infty$ is simply a consequence from scatteringless nature of dimensionally reduced massless fermions, and this kinematical argument is certainly correct. Indeed, such a picture of dimensionally reduced massless fermions provides us with a classical description of the origin of the chiral anomaly [53]. It is essentially important to emphasize that the quantum anomaly is encoded in the Dirac equation in the presence of gauge fields, so that we can in principle implement contributions from the chiral anomaly in our current calculations. Then, the diverging current induced by the topological effect from the LLL is scattered off with higher Landau levels (corresponding to the effect of the relaxation time in refs. [5, 6]) leading to a finite value of $\sigma_{\|}$even in the massless limit. 


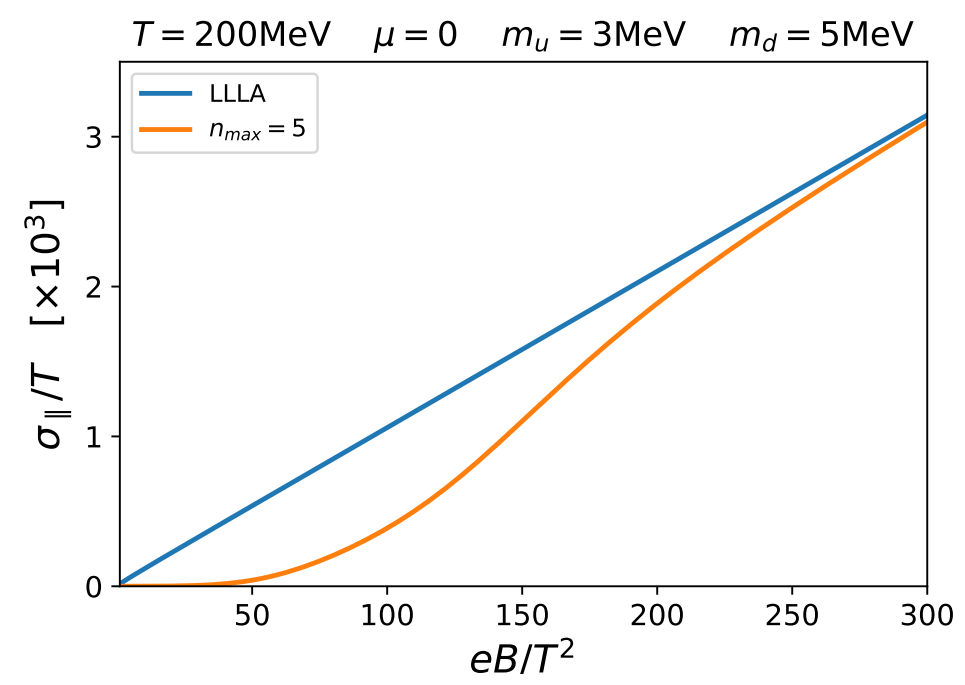

Figure 8. Magnetic field dependence of the longitudinal electric conductivity up to an asymptotically strong field region. The full results (orange color) slowly approaches the results from the LLLA (blue color).

\section{Diagrammatic approach}

Our calculation procedures in section 4 might have looked quite different from direct application of the Kubo formula in section 3. There, in the beginning of section 4, we gave handwaving arguments to relate the Boltzmann equations and the Kubo formula through resummation, and the purpose of this section is spelling out all the algebras to establish this relation in a way as explicit as possible.

In the diagrammatic approach we need to solve the Bethe-Salpeter equations and perform resummation for the propagator and the vertices as illustrated in figure 3 . We here limit our consideration to the zero density $(\mu=0)$ and the single flavor $(q=e)$ case for notational brevity (dropping the flavor index $f$ throughout this section). If necessary, the generalization for nonzero density and/or multiple flavors would be not so difficult. Since we should deal with the pinch singularities caused by the product of retarded and advanced propagators, it would be convenient to employ the " $R / A$ basis" in the real-time formalism $[47,54,55]$. With the $R / A$ basis we can easily identify the pinch singularities out of the diagrams.

\section{$5.1 \quad R / A$ basis}

We can express the quark propagators, $S_{a b}(p)$ in the Schwinger-Keldysh $1 / 2$ basis, appearing in eq. (3.1) and nearby equations, in a matrix product form. We can also represent the gluon propagators, $G_{a b ; \mu \nu}$ in the same way, and they read [47]:

$$
\begin{aligned}
S_{a b}(p) & =U_{a \alpha}\left(p_{0}\right) U_{b \beta}\left(-p_{0}\right) S_{\alpha \beta}(p), \\
G_{a b ; \mu \nu}(k) & =V_{a \alpha}\left(k_{0}\right) V_{b \beta}\left(-k_{0}\right) G_{\alpha \beta ; \mu \nu}(k),
\end{aligned}
$$


with

$$
S_{\alpha \beta}(p)=\left(\begin{array}{cc}
0 & -\mathrm{i} S_{R}(p) \\
-\mathrm{i} S_{A}(p) & 0
\end{array}\right), \quad G_{\alpha \beta ; \mu \nu}=\left(\begin{array}{cc}
0 & -\mathrm{i} G_{R \mu \nu}(k) \\
-\mathrm{i} G_{A \mu \nu}(k) & 0
\end{array}\right) .
$$

Here, the Latin $(a, b=1,2)$ and Greek $(\alpha, \beta=R, A)$ indices represent the components in the $1 / 2$ and the $R / A$ bases, respectively. The transformation matrices are

$$
U_{a \alpha}\left(p_{0}\right)=\left(\begin{array}{cc}
-n_{F}\left(p_{0}\right) & -\mathrm{e}^{-\beta p_{0}} \\
-n_{F}\left(p_{0}\right) & 1
\end{array}\right), \quad V_{a \alpha}\left(k_{0}\right)=\left(\begin{array}{cc}
n_{B}\left(k_{0}\right) & \mathrm{e}^{-\beta k_{0}} \\
n_{B}\left(k_{0}\right) & 1
\end{array}\right) .
$$

Now, the propagators are written in terms of the retarded and the advanced ones, so that we can easily identify the pinch singularities in the diagram. Although the propagator matrix takes such a simple form, the vertices are a little bit complicated. We list up the Feynman rules relevant for our present calculation below. The quark (straight line) and the gluon (curly line) propagators are as simple as

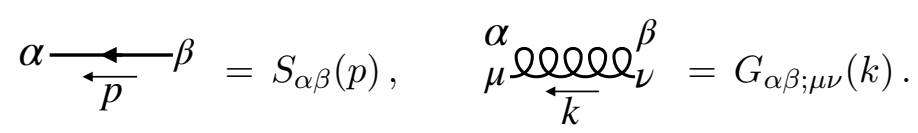

The quark-gluon (including the coupling constant $g$ ) and the quark-photon (excluding the charge $q$ ) vertices involve the Fermi-Dirac distribution function $n_{F}$ and the Bose-Einstein distribution function $n_{B}$ for the $R$ components as

$$
\begin{aligned}
& \overbrace{\alpha_{2}}^{\mu_{k_{2}}^{\alpha_{1}}} \underset{\mathbb{k}_{3} \alpha_{3}}{\longleftarrow}=\mathrm{i} g_{\alpha_{1} \alpha_{2} \alpha_{3}}\left(k_{1}, k_{2}, k_{3}\right) \gamma^{\mu} t^{a} \\
& =\mathrm{i} g \gamma^{\mu} t^{a}\left\{\left[1+n_{B}\left(k_{10}\right)\right]^{\delta_{\alpha_{1}, R}}\left[1-n_{F}\left(k_{20}\right)\right]^{\delta_{\alpha_{2}, R}}\left[1-n_{F}\left(k_{30}\right)\right]^{\delta_{\alpha_{3}, R}}\right. \\
& \left.-\left[n_{B}\left(k_{10}\right)\right]^{\delta_{\alpha_{1}, R}}\left[-n_{F}\left(k_{20}\right)\right]^{\delta_{\alpha_{2}, R}}\left[-n_{F}\left(k_{30}\right)\right]^{\delta_{\alpha_{3}, R}}\right\}, \\
& \overbrace{\alpha_{2}}^{\alpha_{k_{2}}} \underset{\gamma_{k_{3}} \alpha_{3}}{\longleftarrow}=\Gamma_{\alpha_{1} \alpha_{2} \alpha_{3}}^{(0) \mu}\left(k_{1}, k_{2}, k_{3}\right) \\
& =\gamma^{\mu}\left\{\left[1+n_{B}\left(k_{10}\right)\right]^{\delta_{\alpha_{1}, R}}\left[1-n_{F}\left(k_{20}\right)\right]^{\delta_{\alpha_{2}, R}}\left[1-n_{F}\left(k_{30}\right)\right]^{\delta_{\alpha_{3}, R}}\right. \\
& \left.-\left[n_{B}\left(k_{10}\right)\right]^{\delta_{\alpha_{1}, R}}\left[-n_{F}\left(k_{20}\right)\right]^{\delta_{\alpha_{2}, R}}\left[-n_{F}\left(k_{30}\right)\right]^{\delta_{\alpha_{3}, R}}\right\} \text {. }
\end{aligned}
$$

Here, $k_{10}, k_{20}, k_{30}$ denote the zeroth components (i.e., energies) of $k_{1}, k_{2}, k_{3}$, respectively.

\subsection{Expansion of the dressed propagator}

The dressed propagator $S_{\alpha \beta}$ with insertion of self-energy $\Sigma_{\alpha \beta}$ satisfies the following recursive equation:

$$
S_{\alpha \beta}=S_{\alpha \beta}^{(0)}+S_{\alpha \alpha^{\prime}}^{(0)} \Sigma_{\alpha^{\prime} \beta^{\prime}} S_{\beta^{\prime} \beta}
$$


where $S_{\alpha \beta}^{(0)}$ represents the free propagator in the presence of magnetic field. For the retarded propagator, $S_{R}=\mathrm{i} S_{R A}$, the above equation reduces to

$$
S_{R}=S_{R}^{(0)}-S_{R}^{(0)} \Sigma_{R} S_{R}
$$

with $\Sigma_{R}:=\mathrm{i} \Sigma_{A R}$. We can equivalently rewrite this into a more familiar form as

$$
\left(S_{R}^{(0)-1}+\Sigma_{R}\right) S_{R}=1 .
$$

Similarly, the dressed advanced propagator should satisfy, $S_{A}=S_{A}^{(0)}-S_{A}^{(0)} \Sigma_{A} S_{A}$.

We are going to solve eq. (5.9) perturbatively treating $\Sigma_{R}$ as small perturbation. At the zeroth order of the expansion in terms of $\Sigma_{R}$, eq. (5.9) is simply $S_{R}^{(0)-1} S_{R}=1$. For convenience of algebraic transformations, let us introduce the complete basis as defined with the Hamiltonian, $\gamma^{0} S^{(0)-1}=-\mathrm{i} \partial_{0}+H$. The eigenvalue equation reads:

$$
\gamma^{0} S^{(0)-1}|\boldsymbol{n}\rangle=\lambda_{\boldsymbol{n}}|\boldsymbol{n}\rangle,
$$

where $\boldsymbol{n}$ collectively represents a set of all quantum numbers, i.e., $\boldsymbol{n}=\left(p_{0}, p_{z}, t, n, \ell, s\right)$, and the eigenvalue is $\lambda_{\boldsymbol{n}}=-p_{0}+t \varepsilon_{n}$ with $\varepsilon_{n}=\sqrt{p_{z}^{2}+2 e B n+m^{2}}$. In the present notation $t$ is the quantum number for particle $(t=+1)$ and antiparticle $(t=-1), s$ is the spin, and $\ell$ represents other degrees of freedom characterizing the eigenstates which depend on the gauge choice. In the symmetric gauge $\ell$ amounts to the angular momentum $l$ and the color $c$ as we considered for numerical calculations. We normalize the eigenstates as

$$
\left\langle\boldsymbol{n} \mid \boldsymbol{n}^{\prime}\right\rangle=\delta_{\boldsymbol{n}, \boldsymbol{n}^{\prime}}=(2 \pi)^{2} \delta^{(2)}\left(p_{\|}-p_{\|}^{\prime}\right) \delta_{n, n^{\prime}} \delta_{\ell, \ell^{\prime}} \delta_{s, s^{\prime}} \delta_{t, t^{\prime}}
$$

The configuration space representation of states is

$$
\langle x \mid \boldsymbol{n}\rangle=\mathrm{e}^{-\mathrm{i} p_{\|} \cdot x_{\|}}\left[\frac{\delta_{t,+}}{\sqrt{2 \varepsilon_{n}}} u\left(p_{z}, n, \ell, s, \boldsymbol{x}_{\perp}\right)+\frac{\delta_{t,-}}{\sqrt{2 \varepsilon_{n}}} v\left(-p_{z}, n, \ell, s, \boldsymbol{x}_{\perp}\right)\right] .
$$

Here, as usual, $u\left(p_{z}, n, \ell, s, \boldsymbol{x}_{\perp}\right)$ and $v\left(-p_{z}, n, \ell, s, \boldsymbol{x}_{\perp}\right)$ are wave functions of positive and negative energy states solved from

$$
\begin{aligned}
H \mathrm{e}^{\mathrm{i} p_{z} z} u\left(p_{z}, n, \ell, \boldsymbol{x}_{\perp}\right) & =\varepsilon_{n} \mathrm{e}^{\mathrm{i} p_{z} z} u\left(p_{z}, n, \ell, \boldsymbol{x}_{\perp}\right), \\
H \mathrm{e}^{\mathrm{i} p_{z} z} v\left(-p_{z}, n, \ell, \boldsymbol{x}_{\perp}\right) & =-\varepsilon_{n} \mathrm{e}^{\mathrm{i} p_{z} z} v\left(-p_{z}, n, \ell, \boldsymbol{x}_{\perp}\right) .
\end{aligned}
$$

Using these notations we can construct the retarded propagator as

$$
\left.S_{R}^{(0)}\left(x, x^{\prime}\right)=\sum_{\boldsymbol{n}}\langle x \mid \boldsymbol{n}\rangle \frac{1}{\lambda_{\boldsymbol{n}}-\mathrm{i} \epsilon} \overline{\langle\boldsymbol{n}|} x^{\prime}\right\rangle,
$$

where we introduced $\left.\overline{\langle\boldsymbol{n}|} x^{\prime}\right\rangle:=\left\langle\boldsymbol{n} \mid x^{\prime}\right\rangle \gamma^{0}$. Generally $S_{R}^{(0)}\left(x, x^{\prime}\right)$ is not a function of $x-x^{\prime}$ though the magnetic field does not break translational invariance. It is well-known that, summing over $\ell$ and $s$, we can factorize $S_{R}^{(0)}\left(x, x^{\prime}\right)$ as

$$
S_{R}^{(0)}\left(x, x^{\prime}\right)=e^{\mathrm{i} \Theta\left(x, x^{\prime}\right)} \tilde{S}_{R}^{(0)}\left(x-x^{\prime}\right)
$$


into $\tilde{S}_{R}^{(0)}\left(x-x^{\prime}\right)$ [whose Fourier transform is found in eq. (3.5)] and $\Theta\left(x, x^{\prime}\right)$ is called the Schwinger phase. This phase plays no role, which is the case for our present problem, unless we consider operators involving derivative coupling. Therefore, we do not have to take care of the phase that cancels out in the end, and drop the tilde from the propagator for notational brevity. For this translationally invariant part of the propagator we can move to Fourier space in which the propagator takes a form of

$$
\left.\left\langle p\left|S_{R}^{(0)}\right| p^{\prime}\right\rangle=S_{R}^{(0)}\left(p, p^{\prime}\right)=S_{R}^{(0)}(p)(2 \pi)^{4} \delta^{(4)}\left(p-p^{\prime}\right)=\sum_{\boldsymbol{n}}\langle p \mid \boldsymbol{n}\rangle \frac{1}{\lambda_{\boldsymbol{n}}-\mathrm{i} \epsilon} \overline{\langle\boldsymbol{n}|} p^{\prime}\right\rangle,
$$

where

$$
\begin{aligned}
\left\langle p^{\prime} \mid \boldsymbol{n}\right\rangle & =\int \mathrm{d}^{4} x \mathrm{e}^{\mathrm{i} p^{\prime} \cdot x}\langle x \mid \boldsymbol{n}\rangle \\
& =(2 \pi)^{2} \delta^{(2)}\left(p_{\|}-p_{\|}^{\prime}\right) \sqrt{V_{\perp}}\left[\frac{\delta_{t,+}}{\sqrt{2 \varepsilon_{n}}} u\left(\boldsymbol{p}^{\prime}, n, \ell, s\right)+\frac{\delta_{t,-}}{\sqrt{2 \varepsilon_{n}}} v\left(-\boldsymbol{p}^{\prime}, n, \ell, s\right)\right]
\end{aligned}
$$

with Fourier transformed wave functions given as

$$
\begin{aligned}
u(\boldsymbol{p}, n, \ell) & :=\frac{1}{\sqrt{V_{\perp}}} \int \mathrm{d}^{2} x_{\perp} \mathrm{e}^{-\mathrm{i} \boldsymbol{x}_{\perp} \cdot \boldsymbol{p}_{\perp}} u\left(p_{z}, n, \ell, \boldsymbol{x}_{\perp}\right), \\
v(-\boldsymbol{p}, n, \ell) & :=\frac{1}{\sqrt{V_{\perp}}} \int \mathrm{d}^{2} x_{\perp} \mathrm{e}^{-\mathrm{i} \boldsymbol{x}_{\perp} \cdot \boldsymbol{p}_{\perp}} v\left(-p_{z}, n, \ell, \boldsymbol{x}_{\perp}\right) .
\end{aligned}
$$

Here, $V_{\perp}$ is the transverse area on a plane perpendicular to the magnetic field, and $1 / \sqrt{V_{\perp}}$ is the normalization such that the Dirac structures satisfy $\sum_{l, s} u(p) \bar{u}(p)=S_{n}^{f}(p)$ and $\sum_{l, s} v(p) \bar{v}(p)=-S_{n}^{f}(-p)$. We utilized these wave functions in section 4.1.

At the first order of the expansion the leading correction to the propagator is a shift of the eigenvalue given by $\Sigma_{R n}\left(p_{\|}\right):=\left\langle\boldsymbol{n}\left|\Sigma_{R}\right| \boldsymbol{n}\right\rangle$. Precisely speaking, we need to diagonalize the matrix, $\left\langle p_{0}, p_{z}, t, n, \ell, s\left|\Sigma_{R}\right| p_{0}, p_{z}, t, n, \ell^{\prime}, s^{\prime}\right\rangle$ because of degeneracy of Landau levels. Here, we assume a convention after diagonalization, so that the matrix has diagonal components independent of $\ell$ and $s$. Then, we can write the propagator up to the first order as

$$
\left.S_{R}\left(p, p^{\prime}\right)=S_{R}(p)(2 \pi)^{4} \delta^{(4)}\left(p-p^{\prime}\right)=\sum_{\boldsymbol{n}}\langle p \mid \boldsymbol{n}\rangle \frac{1}{\lambda_{\boldsymbol{n}}+\Sigma_{R n}\left(p_{\|}\right)} \overline{\langle\boldsymbol{n}|} p^{\prime}\right\rangle .
$$

The same expression holds for the advanced propagator with $\Sigma_{R n}\left(p_{\|}\right)$replaced with $\Sigma_{A n}\left(p_{\|}\right)$.

\subsection{Resummation of the vertex function and the linearized Boltzmann equa- tions}

We are ready to calculate the longitudinal conductivity from the Kubo formula or more specifically eq. (2.1). In the same way as in eq. (3.22), in the present calculation, we can slightly simplify eq. (2.1) as

$$
\sigma_{\|}=\lim _{k_{0} \rightarrow 0} \lim _{\boldsymbol{k} \rightarrow \mathbf{0}} \frac{1}{\mathrm{i} k_{0}} \operatorname{Im} \Pi_{R}^{33}(k) .
$$

Now, we need to evaluate $\Pi_{R}^{\mu \nu}(k)$ which involves pinch singularities as we explicitly see here. 
The polarization tensor (3.18) at the one loop level can be generalized to the following nonperturbative form:

$\Pi_{R}^{\mu \nu}(k)=(-1) \mathrm{i} q^{2} \int \frac{\mathrm{d}^{4} p}{(2 \pi)^{4}} \operatorname{tr}\left[\Gamma_{A \beta \alpha}^{(0) \mu}(-k,-p, p+k) S^{\alpha \alpha^{\prime}}(p+k) \Gamma_{R \alpha^{\prime} \beta^{\prime}}^{\nu}(k,-p-k, p) S^{\beta^{\prime} \beta}(p)\right]$,

where $\Gamma_{R / A \alpha \beta}^{(0) \mu}$ and $\Gamma_{R / A \alpha \beta}^{\mu}$ are the bare and the dressed retarded/advanced vertex functions. As discussed in the beginning of section 4, the product of retarded and advanced propagators, $S_{R}(p) S_{A}(p)$, causes infrared enhancement due to pinch singularities. We pick this combination of $S_{R}(p) S_{A}(p)$ from the diagram, so that we can approximate $\Pi_{R}^{\mu \nu}(k)$ as

$$
\begin{aligned}
\Pi_{R}^{\mu \nu}(k) \simeq & (-1) \mathrm{i} q^{2}(-\mathrm{i})^{2} \int \frac{\mathrm{d}^{4} p}{(2 \pi)^{4}} \operatorname{tr}\left[\Gamma_{A R R}^{(0) \mu}(-k,-p, p+k) S_{R}(p+k) \Gamma_{R A A}^{\nu}(k,-p-k, p) S_{A}(p)\right] \\
= & \left(\mathrm{e}^{\beta k_{0}}-1\right) \mathrm{i} q^{2} \int \frac{\mathrm{d}^{4} p}{(2 \pi)^{4}} n_{F}\left(p_{0}+k_{0}\right)\left[1-n_{F}\left(p_{0}\right)\right] \\
& \times \operatorname{tr}\left[\gamma^{\mu} S_{R}(p+k) \Gamma_{R A A}^{\nu}(k,-p-k, p) S_{A}(p)\right] .
\end{aligned}
$$

Here, we substituted,

$$
\Gamma_{A R R}^{(0) \mu}\left(k_{1}, k_{2}, k_{3}\right)=\left(\mathrm{e}^{-\beta k_{10}}-1\right) n_{F}\left(k_{20}\right) n_{F}\left(k_{30}\right) \gamma^{\mu},
$$

deduced from eq. (5.6). Now, in this basis, we can easily arrive at the following form of the expression for the longitudinal conductivity:

$$
\sigma_{\|} \simeq \beta q^{2} \operatorname{Re} \int \frac{\mathrm{d}^{4} p}{(2 \pi)^{4}} n_{F}\left(p_{0}\right)\left[1-n_{F}\left(p_{0}\right)\right] \operatorname{tr}\left[\gamma^{3} S_{R}(p) \Gamma^{3}(p) S_{A}(p)\right]
$$

where we introduced $\Gamma^{\mu}(p):=\Gamma_{R A A}^{\nu}(0,-p, p)$ to simplify the notation.

We need to evaluate $S_{R}(p) \Gamma^{3}(p) S_{A}(p)$, in which both propagator and the vertex functions are dressed. We shall first consider the case with $\Gamma^{3}(p)=\gamma^{3}$ to see the behavior of the pinch singularity. Using eq. (5.19), we can write down an explicit integral of $S_{R}(p) \gamma^{3} S_{A}(p)$ as

$$
\begin{aligned}
& S_{R}(p) \gamma^{3} S_{A}(p)=\int \frac{\mathrm{d}^{4} p^{\prime}}{(2 \pi)^{4}} \int \frac{\mathrm{d}^{4} p^{\prime \prime}}{(2 \pi)^{4}}(2 \pi)^{4} \delta^{(4)}\left(p-p^{\prime}\right)(2 \pi)^{4} \delta^{(4)}\left(p^{\prime}-p^{\prime \prime}\right) S_{R}(p) \gamma^{3} S_{A}\left(p^{\prime}\right) \\
& \left.\left.=\sum_{\boldsymbol{n}, \boldsymbol{n}^{\prime}} \int \frac{\mathrm{d}^{4} p^{\prime}}{(2 \pi)^{4}} \int \frac{\mathrm{d}^{4} p^{\prime \prime}}{(2 \pi)^{4}}\langle p \mid \boldsymbol{n}\rangle \frac{1}{\lambda_{\boldsymbol{n}}+\Sigma_{R n}\left(p_{\|}\right)} \overline{\langle\boldsymbol{n}|} p^{\prime}\right\rangle \gamma^{3}\left\langle p^{\prime} \mid \boldsymbol{n}^{\prime}\right\rangle \frac{1}{\lambda_{\boldsymbol{n}^{\prime}}+\Sigma_{A n^{\prime}}\left(p_{\|}\right)} \overline{\left\langle\boldsymbol{n}^{\prime}\right|} p^{\prime \prime}\right\rangle .
\end{aligned}
$$

With the wave functions (5.17) we can evaluate the matrix element as

$$
\left.\int \frac{\mathrm{d}^{4} p^{\prime}}{(2 \pi)^{4}} \overline{\langle\boldsymbol{n}|} p^{\prime}\right\rangle \gamma^{3}\left\langle p^{\prime} \mid \boldsymbol{n}^{\prime}\right\rangle=\overline{\langle\boldsymbol{n}|} \gamma^{3}\left|\boldsymbol{n}^{\prime}\right\rangle=\frac{p_{z}}{\varepsilon_{n}} \delta_{\boldsymbol{n}, \boldsymbol{n}^{\prime}}+\cdots
$$

where the ellipsis, $\cdots$, denotes the off-diagonal components with $\boldsymbol{n} \neq \boldsymbol{n}^{\prime}$. We note that $p_{z}$ is a momentum contained in $\boldsymbol{n}$, but with $\langle p \mid \boldsymbol{n}\rangle$, we can identify $p_{z}$ as the same one in the argument of $S_{R / A}(p)$ [see eq. (5.17)]. These off-diagonal components do not contribute 
to the pinch singularities, and thus, we can safely neglect them. We eventually simplify $S_{R}(p) \gamma^{3} S_{A}(p)$ dropping the off-diagonal components as

$$
\left.S_{R}(p) \gamma^{3} S_{A}(p) \simeq \sum_{\boldsymbol{n}} \int \frac{\mathrm{d}^{4} p^{\prime \prime}}{(2 \pi)^{4}} \frac{p_{z}}{\varepsilon_{n}}\langle p \mid \boldsymbol{n}\rangle \frac{1}{\lambda_{\boldsymbol{n}}+\Sigma_{R n}\left(p_{\|}\right)} \cdot \frac{1}{\lambda_{\boldsymbol{n}}+\Sigma_{A n}\left(p_{\|}\right)} \overline{\langle\boldsymbol{n}|} p^{\prime \prime}\right\rangle .
$$

We note that from the spectral function (3.12) the following relation holds,

$$
\begin{aligned}
& \frac{1}{-2 \operatorname{Im} \Sigma_{R n}\left(p_{\|}\right)} S_{n}(p)(2 \pi) \operatorname{sgn}\left(p_{0}\right) \delta\left(p_{0}^{2}-\varepsilon_{n}^{2}\right)(2 \pi)^{4} \delta\left(p-p^{\prime \prime}\right) \\
& \left.\simeq \sum_{\boldsymbol{n}}{ }^{\prime}\langle p \mid \boldsymbol{n}\rangle \frac{1}{\left[\lambda_{\boldsymbol{n}}+\Sigma_{R n}\right]\left[\lambda_{\boldsymbol{n}}+\Sigma_{A n}\right]} \overline{\langle\boldsymbol{n}|} p^{\prime \prime}\right\rangle
\end{aligned}
$$

apart from the energy shift by $\Sigma_{R / A n}$ of higher order. Here, the prime denotes the sum over $\boldsymbol{n}$ except $n$. Substituting this into the above, we can find the following form:

$$
S_{R}(p) \gamma^{3} S_{A}(p)=\sum_{n} \frac{p_{z}}{\varepsilon_{n}} \cdot \frac{1}{-2 \operatorname{Im} \Sigma_{R n}\left(p_{\|}\right)} S_{n}(p)(2 \pi) \operatorname{sgn}\left(p_{0}\right) \delta\left(p_{0}^{2}-\varepsilon_{n}^{2}\right) .
$$

It is clear from this form that the weak coupling limit, $\operatorname{Im} \Sigma_{R n}\left(p_{\|}\right) \rightarrow 0$, makes $S_{R}(p) \gamma^{3} S_{A}(p)$ diverge, which is nothing but the pinch singularity. We also note that $\overline{\langle\boldsymbol{n}|} \gamma^{1,2}\left|\boldsymbol{n}^{\prime}\right\rangle$ do not have the diagonal component, so that no pinch singularity appears in the transverse and Hall conductivities, which justifies our calculations in section 3 .

The above exercise of $S_{R}(p) \gamma^{3} S_{A}(p)$ implies the following reasonable Ansatz for the Dirac structure of the vertex function as

$$
S_{R}(p) q \Gamma^{3}(p) S_{A}(p)=\sum_{n} \tilde{\chi}\left(p_{\|}\right) S_{n}(p)(2 \pi) \operatorname{sgn}\left(p_{0}\right) \delta\left(p_{0}^{2}-\varepsilon_{n}^{2}\right)
$$

We note that $\tilde{\chi}\left(p_{\|}\right)$is also an $n$ dependent function in an implicit way through $p_{0}$. With this form we can treat the transverse momentum integral with $\operatorname{tr}\left[\gamma^{3} S_{n}(p)\right]$ in the same way as in eq. (3.15), and then the longitudinal conductivity looks,

$$
\sigma_{\|}=\beta N_{\mathrm{c}} \frac{q^{2} B}{2 \pi} \sum_{n} \alpha_{n} \int \frac{\mathrm{d}^{2} p_{\|}}{(2 \pi)^{2}} 2 p_{z} n_{F}\left(p_{0}\right)\left[1-n_{F}\left(p_{0}\right)\right](2 \pi) \operatorname{sgn}\left(p_{0}\right) \delta\left(p_{0}^{2}-\varepsilon_{n}^{2}\right) \tilde{\chi}\left(p_{\|}\right) .
$$

We can finally perform the $p_{0}$ integration to find,

$$
\sigma_{\|}=\beta N_{\mathrm{c}} \frac{q^{2} B}{2 \pi} \sum_{n} \alpha_{n} \int \frac{\mathrm{d} p_{z}}{2 \pi} \frac{p_{z}}{\varepsilon_{n}} n_{F}\left(\varepsilon_{n}\right)\left[1-n_{F}\left(\varepsilon_{n}\right)\right]\left(\chi_{p}-\bar{\chi}_{p}\right),
$$

where we introduced a notation, $\chi_{p}=\tilde{\chi}\left(\varepsilon_{n}, p_{z}\right)$ and $\overline{\chi_{p}}=-\tilde{\chi}\left(-\varepsilon_{n},-p_{z}\right)$. This final expression exactly corresponds to the second line of eq. (4.16).

The remaining task is to derive a condition to fix $\chi_{p}$ and $\bar{\chi}_{p}$, which needs the determination of the vertex function. At the leading order the Bethe-Salpeter equation shown 
in figure 3 reads:

$$
\begin{aligned}
\Gamma^{\mu}(p)= & \gamma^{\mu}+(\mathrm{i})^{2} \int \frac{\mathrm{d}^{4} p^{\prime}}{(2 \pi)^{4}} g_{\gamma A \alpha}\left(k,-p, p^{\prime}\right) t^{a} g_{\gamma^{\prime} \beta A}\left(-k,-p^{\prime}, p\right) t^{a} G_{\rho \nu}^{\gamma \gamma^{\prime}}(k) \\
& \times \gamma^{\rho} S^{\alpha \alpha^{\prime}}\left(p^{\prime}\right) \Gamma_{R \alpha^{\prime} \beta^{\prime}}^{\mu}\left(0,-p^{\prime}, p^{\prime}\right) S^{\beta^{\prime} \beta}\left(p^{\prime}\right) \gamma^{\nu} \\
\simeq & \gamma^{\mu}+C_{F} \int \frac{\mathrm{d}^{4} p^{\prime}}{(2 \pi)^{4}} g_{\gamma A R}\left(k,-p, p^{\prime}\right) g_{\gamma^{\prime} R A}\left(-k,-p^{\prime}, p\right) G_{\rho \nu}^{\gamma \gamma^{\prime}}(k) \gamma^{\rho} S_{R}\left(p^{\prime}\right) \Gamma^{\mu}\left(p^{\prime}\right) S_{A}\left(p^{\prime}\right) \gamma^{\nu},
\end{aligned}
$$

where $k=p-p^{\prime}$ and $C_{F}=\left(N_{\mathrm{c}}^{2}-1\right) /\left(2 N_{\mathrm{c}}\right)$. From the first to the second lines the approximation symbol, $\simeq$, indicates a prescription to pick up the pinch singularity only. From the Feynman rule (5.5), the product of the coupling can be evaluated as

$$
\begin{aligned}
& g_{R A R}\left(k,-p, p^{\prime}\right) g_{A R A}\left(-k,-p^{\prime}, p\right)=g^{2}\left\{\left[1+n_{B}\left(k_{0}\right)\right]\left[1-n_{F}\left(p_{0}^{\prime}\right)\right]+n_{B}\left(k_{0}\right) n_{F}\left(p_{0}^{\prime}\right)\right\}, \\
& g_{R R A}\left(k,-p, p^{\prime}\right) g_{A A R}\left(-k,-p^{\prime}, p\right)=-g^{2}\left\{\left[1+n_{B}\left(k_{0}\right)\right]\left[1-n_{F}\left(p_{0}^{\prime}\right)\right]+n_{B}\left(k_{0}\right) n_{F}\left(p_{0}^{\prime}\right)\right\} .
\end{aligned}
$$

Then, these expressions reduce the Bethe-Salpeter equation into a simple form as

$$
\begin{aligned}
\gamma^{\mu}= & \Gamma^{\mu}(p)-\frac{g^{2} C_{F}}{n_{F}\left(p_{0}\right)\left[1-n_{F}\left(p_{0}\right)\right]} \int \frac{\mathrm{d}^{4} p^{\prime}}{(2 \pi)^{4}}\left[1+n_{B}\left(k_{0}\right)\right]\left[1-n_{F}\left(p_{0}^{\prime}\right)\right] n_{F}\left(p_{0}\right) \rho_{\rho \nu}(k) \\
& \times \gamma^{\rho} S_{R}\left(p^{\prime}\right) \Gamma^{\mu}\left(p^{\prime}\right) S_{A}\left(p^{\prime}\right) \gamma^{\nu} .
\end{aligned}
$$

Here, in the above, we interchanged $\Gamma^{\mu}$ and $\gamma^{\mu}$ from the left- and the right-hand sides for convenience and flipped the sign. The gluon propagator amounts to the spectral function, $\rho_{\rho \nu}(k):=G_{\nu \rho}^{R A}(k)-G_{\nu \rho}^{A R}(k)=-\mathrm{i}\left[G_{R \nu \rho}(k)-G_{A \nu \rho}(k)\right]$. At the leading order of coupling constant, in the Feynman gauge, the spectral function reads,

$$
\rho_{\rho \nu}(k)=-\eta_{\rho \nu}(2 \pi) \operatorname{sgn}\left(k_{0}\right) \delta\left(k^{2}\right) .
$$

Then, we aim to translate the condition (5.35) for $\chi_{p}$ and $\bar{\chi}_{p}$ contained in $\Gamma^{\mu}\left(p^{\prime}\right)$ into the form of linearized Boltzmann equations. The explicit relation between the vertex function and $\tilde{\chi}$ is given by eq. (5.30), from which we can easily solve $\Gamma^{3}\left(p^{\prime}\right)$ as

$$
\begin{aligned}
q \Gamma^{3}\left(p^{\prime}\right) & =S_{R}\left(p^{\prime}\right)^{-1} \sum_{n} \tilde{\chi}\left(p_{\|}^{\prime}\right) S_{n^{\prime}}\left(p^{\prime}\right)(2 \pi) \operatorname{sgn}\left(p_{0}^{\prime}\right) \delta\left(p_{0}^{\prime 2}-\varepsilon_{n^{\prime}}^{2}\right) S_{A}\left(p^{\prime}\right)^{-1} \\
& =-\mathrm{i}\left[S_{A}\left(p^{\prime}\right)^{-1}-S_{R}\left(p^{\prime}\right)^{-1}\right] \tilde{\chi}\left(p_{\|}\right) \\
& =-2 \operatorname{Im} \Sigma(p) \tilde{\chi}\left(p_{\|}\right) .
\end{aligned}
$$

Multiplying the Bethe-Salpeter equation (5.35) by $q S_{n}(p)$, taking the trace, and using eqs. (5.30), (5.36), and (5.37), we find,

$$
\begin{aligned}
& \left.q N_{\mathrm{c}} \operatorname{tr}\left[S_{n}(p) \gamma^{3}\right]=-N_{\mathrm{c}} \operatorname{tr}\left[S_{n}(p) 2 \operatorname{Im} \Sigma_{R}(p)\right)\right] \tilde{\chi}\left(p_{\|}\right) \\
& \quad-\frac{g^{2} C_{F} N_{\mathrm{c}}}{n_{F}\left(p_{0}\right)\left[1-n_{F}\left(p_{0}\right)\right]} \sum_{n^{\prime}} \int \frac{\mathrm{d}^{4} p^{\prime}}{(2 \pi)^{4}}\left[1+n_{B}\left(k_{0}\right)\right]\left[1-n_{F}\left(p_{0}^{\prime}\right)\right] n_{F}\left(p_{0}\right) \\
& \quad \times(2 \pi) \operatorname{sgn}\left(k_{0}\right) \delta\left(k^{2}\right)(2 \pi) \operatorname{sgn}\left(p_{0}^{\prime}\right) \delta\left(p_{0}^{\prime 2}-\varepsilon_{n^{\prime}}^{2}\right)\left\{-\operatorname{tr}\left[S_{n}(p) \gamma_{\nu} S_{n^{\prime}}\left(p^{\prime}\right) \gamma^{\nu}\right] \tilde{\chi}\left(p_{\|}^{\prime}\right)\right\} .
\end{aligned}
$$


The direct evaluation of the self-energy yields,

$$
\begin{aligned}
2 \operatorname{Im} \Sigma_{R}(p)= & 2 \operatorname{Im}(\mathrm{i})^{3} \int \frac{\mathrm{d}^{4} p^{\prime}}{(2 \pi)^{4}} G_{\alpha^{\prime} \alpha ; \mu \nu}(k) g_{\alpha^{\prime} A \beta^{\prime}}\left(k,-p, p^{\prime}\right) \gamma^{\mu} t^{a} S_{\beta^{\prime} \beta}\left(p^{\prime}\right) g_{\alpha \beta R}\left(-k,-p^{\prime}, p\right) \gamma^{\nu} t^{a} \\
= & 2 \operatorname{Re} C_{F} \int \frac{\mathrm{d}^{4} p^{\prime}}{(2 \pi)^{4}}\left[G_{A \mu \nu}(k) g_{A A R}\left(k,-p, p^{\prime}\right) \gamma^{\mu} S_{R}\left(p^{\prime}\right) g_{R A R}\left(-k,-p^{\prime}, p\right) \gamma^{\nu}\right. \\
& +G_{R \mu \nu}(k) g_{R A A}\left(k,-p, p^{\prime}\right) \gamma^{\mu} S_{A}\left(p^{\prime}\right) g_{A R R}\left(-k,-p^{\prime}, p\right) \gamma^{\nu} \\
& \left.+G_{R \mu \nu}(k) g_{R A R}\left(k,-p, p^{\prime}\right) \gamma^{\mu} S_{R}\left(p^{\prime}\right) g_{A A R}\left(-k,-p^{\prime}, p\right) \gamma^{\nu}\right] \\
= & \frac{g^{2} C_{F}}{n_{F}\left(p_{0}\right)\left[1-n_{F}\left(p_{0}\right)\right]} \sum_{n^{\prime}} \int \frac{\mathrm{d}^{4} p^{\prime}}{(2 \pi)^{4}}\left[1+n_{B}\left(k_{0}\right)\right]\left[1-n_{F}\left(p_{0}^{\prime}\right)\right] n_{F}\left(p_{0}\right) \\
& \times(2 \pi) \operatorname{sgn}\left(k_{0}\right) \delta\left(k^{2}\right)(2 \pi) \operatorname{sgn}\left(p_{0}^{\prime}\right) \delta\left(p_{0}^{\prime 2}-\varepsilon_{n^{\prime}}^{2}\right) \gamma_{\nu} S_{n^{\prime}}\left(p^{\prime}\right) \gamma^{\nu} .
\end{aligned}
$$

Interestingly, this form has similarity to the second term in eq. (5.38), so that we can further simplify the Bethe-Salpeter equation into the following form:

$$
\begin{aligned}
N_{\mathrm{c}} \frac{p_{z}}{p_{0}} \operatorname{tr}\left[\gamma^{0} S_{n}(p)\right]= & -\frac{g^{2} C_{F} N_{\mathrm{c}}}{n_{F}\left(p_{0}\right)\left[1-n_{F}\left(p_{0}\right)\right]} \sum_{n^{\prime}} \int \frac{\mathrm{d}^{4} k}{(2 \pi)^{4}} \int \frac{\mathrm{d}^{4} p^{\prime}}{(2 \pi)^{4}}(2 \pi)^{4} \delta^{(4)}\left(p-k-p^{\prime}\right) \\
& \times \operatorname{tr}\left[S_{n}(p) \gamma_{\nu} S_{n^{\prime}}\left(p^{\prime}\right) \gamma^{\nu}\right] n_{F}\left(p_{0}\right)\left[1-n_{F}\left(p_{0}^{\prime}\right)\right]\left[1+n_{B}\left(k_{0}\right)\right](2 \pi) \operatorname{sgn}\left(k_{0}\right) \delta\left(k^{2}\right) \\
& \times(2 \pi) \operatorname{sgn}\left(p_{0}^{\prime}\right) \delta\left(p_{0}^{\prime 2}-\varepsilon_{n^{\prime}}^{2}\right)\left[\tilde{\chi}\left(p_{\|}\right)-\tilde{\chi}\left(p_{\|}^{\prime}\right)\right],
\end{aligned}
$$

where we employed $\operatorname{tr}\left[\gamma^{3} S_{n}(p)\right]=\left(p_{z} / p_{0}\right) \operatorname{tr}\left[\gamma^{0} S_{n}(p)\right]$ to rewrite the left-hand side.

To confirm that the above expression is nothing but the Boltzmann equation, we shall focus on the positive energy state, i.e., $p_{0}=\varepsilon_{n}>0$. We perform the $k_{0}$ and $p_{0}^{\prime}$ integrations in the left-hand side of eq. (5.40), and then, the expression takes the following form,

$$
\begin{aligned}
N_{\mathrm{c}} & \frac{p_{z}}{\varepsilon_{n}} \operatorname{tr}\left[\gamma^{0} S_{n}(p)\right] \\
= & \frac{1}{n_{F}\left(\varepsilon_{n}\right)\left[1-n_{F}\left(\varepsilon_{n}\right)\right]} \sum_{n^{\prime}} \int \frac{\mathrm{d}^{3} k}{(2 \pi)^{3}} \frac{1}{2|\boldsymbol{k}|} \int \frac{\mathrm{d}^{3} p^{\prime}}{(2 \pi)^{3}} \frac{1}{2 \varepsilon_{n^{\prime}}} \\
& \times\left\{\overline{\left|\mathcal{M}_{p \rightarrow p^{\prime}+k}\right|}(2 \pi)^{4} \delta^{(4)}\left(p-k-p^{\prime}\right) n_{F}\left(\varepsilon_{n}\right)\left[1-n_{F}\left(\varepsilon_{n^{\prime}}\right)\right]\left[1+n_{B}(|\boldsymbol{k}|)\right]\left(\chi_{p}-\chi_{p^{\prime}}\right)\right. \\
& +\overline{\left|\mathcal{M}_{p+k \rightarrow p^{\prime}}\right|}(2 \pi)^{4} \delta^{(4)}\left(p+k-p^{\prime}\right) n_{F}\left(\varepsilon_{n}\right) n_{B}(|\boldsymbol{k}|)\left[1-n_{F}\left(\varepsilon_{n^{\prime}}\right)\right]\left(\chi_{p}-\chi_{p^{\prime}}\right) \\
& \left.+\overline{\left|\mathcal{M}_{p+p^{\prime} \rightarrow k}\right|}(2 \pi)^{4} \delta^{(4)}\left(p-k+p^{\prime}\right) n_{F}\left(\varepsilon_{n}\right) n_{F}\left(\varepsilon_{n^{\prime}}\right)\left[1+n_{B}(|\boldsymbol{k}|)\right]\left(\chi_{p}+\bar{\chi}_{p^{\prime}}\right)\right\} .
\end{aligned}
$$

Here, we defined the squared matrix element averaging over all internal degrees of freedom as

$$
\begin{aligned}
& \overline{\left|\mathcal{M}_{p \rightarrow p^{\prime}+k}\right|}:=-\left.g^{2} C_{F} N_{\mathrm{c}} \operatorname{tr}\left[S_{n}(p) \gamma_{\nu} S_{n^{\prime}}\left(p^{\prime}\right) \gamma^{\nu}\right]\right|_{p_{0}^{\prime}=\varepsilon_{n^{\prime}}, k_{0}=|\boldsymbol{k}|}, \\
& \overline{\left|\mathcal{M}_{p+k \rightarrow p^{\prime}}\right|}:=-\left.g^{2} C_{F} N_{\mathrm{c}} \operatorname{tr}\left[S_{n}(p) \gamma_{\nu} S_{n^{\prime}}\left(p^{\prime}\right) \gamma^{\nu}\right]\right|_{p_{0}^{\prime}=\varepsilon_{n^{\prime}}, k_{0}=|\boldsymbol{k}|}, \\
& \overline{\left|\mathcal{M}_{p+p^{\prime} \rightarrow k}\right|}:=\left.g^{2} C_{F} N_{\mathrm{c}} \operatorname{tr}\left[S_{n}(p) \gamma_{\nu} S_{n^{\prime}}\left(-p^{\prime}\right) \gamma^{\nu}\right]\right|_{p_{0}^{\prime}=\varepsilon_{n^{\prime}}, k_{0}=|\boldsymbol{k}|}
\end{aligned}
$$


Now, we see that eq. (5.41) is nothing but the linearized Boltzmann equation as considered in section 4. This completes our discussions on the diagrammatic derivation of the Boltzmann equations.

\section{Conclusions}

We spelled out all the details about the field-theoretical calculation of the electric conductivity beyond the lowest Landau level approximation. Including higher Landau level contributions the electric conductivity turns out to behave smoothly as a function of fermion mass, and particularly, we found that the electric conductivity remains finite even in the massless limit. The diverging part of the electric conductivity from the lowest Landau level is attributed to the chiral magnetic effect which predicts a finite production rate of topological current, that is, a topological current proportional to time. This current is scattered off with higher Landau levels at finite mass, so that a steady state is balanced with current not blowing up, and we can obtain a physically sensible estimate for the electric conductivity.

Contrary to the common statement that the chiral magnetic effect leads to quadratic suppression of the negative magnetoresistance, our results show that the asymptotic behavior of the electric conductivity is linearly suppressed with increasing magnetic field. In fact, we found that in an intermediate region of the applied magnetic field, there is a window where the magnetic dependence of the electric conductivity looks quadratic approximately. This crucial difference from the previous argument based on the relaxation time approximation appears from potential magnetic dependence of the relaxation time.

Technically speaking, our formulation itself contains some notable features even apart from complications associated with the Landau level sum. We made resummation to deal with the pinch singularities, for which we started with the Bethe-Salpeter equation. The most cumbersome part in the calculations lies in the field-theoretical reduction from the Bethe-Salpeter equation into the Boltzmann equation. In the present paper, we first presented the electric conductivity results presuming the Boltzmann equation, and later on, we gave full calculations based on the Schwinger-Keldysh formalism to justify our kinetic treatments. At some point of our calculations one might have wondered a relation to what has been discussed in the context of the chiral kinetic theory. We note here that, when we refer to the Boltzmann equation in this work, we retain the Dirac matrix structures. In the chiral kinetic theory, in contrast, the Dirac structure or the spin dynamics is encoded in the Berry curvature in the adiabatic approximation. In this sense we can say that nothing is dropped off from our present formulation keeping the Dirac matrices, and the effect of the chiral anomaly must be fully incorporated even though the Berry curvature does not manifest itself here.

We assumed fundamental dynamics of quarks and gluons in this work, but it should be feasible to apply our methodology in order to explain the table-top experiment of the negative magnetoresistance. For this purpose, unlike QCD that is clearly defined by a simple Lagrangian, we need to adopt some impurity model and introduce interactions between gapless fermions and phonons. It would be then a challenging problem to at- 
tempt theoretical calculations in order to understand quantitative features of the negative magnetoresistance such as the detailed shape of magnetic dependence, the temperature dependence of the magnetoresistance, similarity and difference depending on various chiral materials, and so on. More and more exciting developments in theory will be awaiting us, and we believe that the present work takes the initiative in those directions of future research.

\section{Acknowledgments}

The authors thank, Dima Kharzeev, Koichi Hattori, and Daisuke Satow for discussions. This work was supported in part by the ExtreMe Matter Institute EMMI at the GSI Helmholtzzentrum für Schwerionenphysik, Darmstadt, Germany. K. F. is grateful for warm hospitality at Heidelberg University where a part of this work was complete. This work was also supported by Japan Society for the Promotion of Science (JSPS) KAKENHI Grant No. 16K17716, 17H06462, and 18H01211.

Open Access. This article is distributed under the terms of the Creative Commons Attribution License (CC-BY 4.0), which permits any use, distribution and reproduction in any medium, provided the original author(s) and source are credited.

\section{References}

[1] D.E. Kharzeev, L.D. McLerran and H.J. Warringa, The Effects of topological charge change in heavy ion collisions: 'Event by event P and CP-violation', Nucl. Phys. A 803 (2008) 227 [arXiv:0711.0950] [INSPIRE].

[2] K. Fukushima, D.E. Kharzeev and H.J. Warringa, The Chiral Magnetic Effect, Phys. Rev. D 78 (2008) 074033 [arXiv: 0808.3382] [INSPIRE].

[3] M.A. Metlitski and A.R. Zhitnitsky, Anomalous axion interactions and topological currents in dense matter, Phys. Rev. D 72 (2005) 045011 [hep-ph/0505072] [INSPIRE].

[4] K. Fukushima, D.E. Kharzeev and H.J. Warringa, Real-time dynamics of the Chiral Magnetic Effect, Phys. Rev. Lett. 104 (2010) 212001 [arXiv:1002.2495] [INSPIRE].

[5] D.T. Son and B.Z. Spivak, Chiral Anomaly and Classical Negative Magnetoresistance of Weyl Metals, Phys. Rev. B 88 (2013) 104412 [arXiv:1206.1627] [InSPIRE].

[6] Q. Li et al., Observation of the chiral magnetic effect in ZrTe $e_{5}$, Nature Phys. 12 (2016) 550 [arXiv: 1412.6543] [INSPIRE].

[7] D.E. Kharzeev, J. Liao, S.A. Voloshin and G. Wang, Chiral magnetic and vortical effects in high-energy nuclear collisions - A status report, Prog. Part. Nucl. Phys. 88 (2016) 1 [arXiv: 1511.04050] [INSPIRE].

[8] K. Fukushima, Views of the Chiral Magnetic Effect, Lect. Notes Phys. 871 (2013) 241 [arXiv: 1209.5064] [INSPIRE].

[9] J. Xiong et al., Evidence for the chiral anomaly in the Dirac semimetal $N a_{3} B i$, Science $\mathbf{3 5 0}$ (2015) 413. 
[10] C.-Z. Li, L.-X. Wang, H. Liu, J. Wang, Z.-M. Liao and D.-P. Yu, Giant negative magnetoresistance induced by the chiral anomaly in individual $\mathrm{Cd}_{3} A s_{2}$ nanowires, Nature Commun. 6 (2015) 10137 [arXiv: 1504.07398].

[11] X. Huang et al., Observation of the Chiral-Anomaly-Induced Negative Magnetoresistance in $3 D$ Weyl Semimetal TaAs, Phys. Rev. X 5 (2015) 031023 [arXiv:1503.01304] [InSPIRE].

[12] F. Arnold et al., Negative magnetoresistance without well-defined chirality in the Weyl semimetal TaP, Nature Commun. 7 (2016) 1615 [arXiv:1506.06577] [INSPIRE].

[13] G.V. Dunne, Heisenberg-Euler effective Lagrangians: Basics and extensions, in From fields to strings: Circumnavigating theoretical physics. Ian Kogan memorial collection. Vol. 3, M. Shifman, A. Vainshtein and J. Wheater eds., World Scientific, New York U.S.A. (2004), pg. 445 [hep-th/0406216] [INSPIRE].

[14] P. Copinger, K. Fukushima and S. Pu, Axial Ward identity and the Schwinger mechanism Applications to the real-time chiral magnetic effect and condensates, Phys. Rev. Lett. 121 (2018) 261602 [arXiv:1807.04416] [INSPIRE].

[15] K. Fukushima, Extreme matter in electromagnetic fields and rotation, Prog. Part. Nucl. Phys. 107 (2019) 167 [arXiv: 1812.08886] [INSPIRE].

[16] K. Fukushima and Y. Hidaka, Magnetic Shift of the Chemical Freeze-out and Electric Charge Fluctuations, Phys. Rev. Lett. 117 (2016) 102301 [arXiv: 1605. 01912] [INSPIRE].

[17] D.T. Son and N. Yamamoto, Berry Curvature, Triangle Anomalies and the Chiral Magnetic Effect in Fermi Liquids, Phys. Rev. Lett. 109 (2012) 181602 [arXiv:1203.2697] [INSPIRE].

[18] M.A. Stephanov and Y. Yin, Chiral Kinetic Theory, Phys. Rev. Lett. 109 (2012) 162001 [arXiv: 1207.0747] [INSPIRE].

[19] J.-H. Gao, Z.-T. Liang, S. Pu, Q. Wang and X.-N. Wang, Chiral Anomaly and Local Polarization Effect from Quantum Kinetic Approach, Phys. Rev. Lett. 109 (2012) 232301 [arXiv: 1203.0725] [INSPIRE].

[20] D.T. Son and N. Yamamoto, Kinetic theory with Berry curvature from quantum field theories, Phys. Rev. D 87 (2013) 085016 [arXiv:1210.8158] [InSPIRE].

[21] J.-W. Chen, S. Pu, Q. Wang and X.-N. Wang, Berry Curvature and Four-Dimensional Monopoles in the Relativistic Chiral Kinetic Equation, Phys. Rev. Lett. 110 (2013) 262301 [arXiv: 1210.8312] [INSPIRE].

[22] C. Manuel and J.M. Torres-Rincon, Kinetic theory of chiral relativistic plasmas and energy density of their gauge collective excitations, Phys. Rev. D 89 (2014) 096002 [arXiv: 1312.1158] [INSPIRE].

[23] J.-Y. Chen, D.T. Son, M.A. Stephanov, H.-U. Yee and Y. Yin, Lorentz Invariance in Chiral Kinetic Theory, Phys. Rev. Lett. 113 (2014) 182302 [arXiv:1404.5963] [INSPIRE].

[24] J.-Y. Chen, D.T. Son and M.A. Stephanov, Collisions in Chiral Kinetic Theory, Phys. Rev. Lett. 115 (2015) 021601 [arXiv:1502.06966] [INSPIRE].

[25] Y. Hidaka, S. Pu and D.-L. Yang, Relativistic Chiral Kinetic Theory from Quantum Field Theories, Phys. Rev. D 95 (2017) 091901 [arXiv:1612.04630] [INSPIRE].

[26] Y. Hidaka, S. Pu and D.-L. Yang, Nonlinear Responses of Chiral Fluids from Kinetic Theory, Phys. Rev. D 97 (2018) 016004 [arXiv:1710.00278] [INSPIRE]. 
[27] Y. Hidaka and D.-L. Yang, Nonequilibrium chiral magnetic/vortical effects in viscous fluids, Phys. Rev. D 98 (2018) 016012 [arXiv: 1801.08253] [INSPIRE].

[28] N. Mueller and R. Venugopalan, Worldline construction of a covariant chiral kinetic theory, Phys. Rev. D 96 (2017) 016023 [arXiv:1702.01233] [INSPIRE].

[29] N. Mueller and R. Venugopalan, The chiral anomaly, Berry's phase and chiral kinetic theory, from world-lines in quantum field theory, Phys. Rev. D 97 (2018) 051901 [arXiv: 1701.03331] [INSPIRE].

[30] A. Huang, S. Shi, Y. Jiang, J. Liao and P. Zhuang, Complete and Consistent Chiral Transport from Wigner Function Formalism, Phys. Rev. D 98 (2018) 036010 [arXiv: 1801.03640] [INSPIRE].

[31] S. Carignano, C. Manuel and J.M. Torres-Rincon, Consistent relativistic chiral kinetic theory: A derivation from on-shell effective field theory, Phys. Rev. D 98 (2018) 076005 [arXiv: 1806.01684] [INSPIRE].

[32] Ö.F. Dayi and E. Kilinçarslan, Quantum Kinetic Equation in the Rotating Frame and Chiral Kinetic Theory, Phys. Rev. D 98 (2018) 081701 [arXiv:1807.05912] [INSPIRE].

[33] Y.-C. Liu, L.-L. Gao, K. Mameda and X.-G. Huang, Chiral kinetic theory in curved spacetime, Phys. Rev. D 99 (2019) 085014 [arXiv:1812.10127] [INSPIRE].

[34] S. Lin and A. Shukla, Chiral Kinetic Theory from Effective Field Theory Revisited, JHEP 06 (2019) 060 [arXiv: 1901.01528] [INSPIRE].

[35] K. Fukushima, K. Hattori, H.-U. Yee and Y. Yin, Heavy Quark Diffusion in Strong Magnetic Fields at Weak Coupling and Implications for Elliptic Flow, Phys. Rev. D 93 (2016) 074028 [arXiv: 1512.03689] [INSPIRE].

[36] K. Hattori and D. Satow, Electrical Conductivity of Quark-Gluon Plasma in Strong Magnetic Fields, Phys. Rev. D 94 (2016) 114032 [arXiv:1610.06818] [InSPIRE].

[37] K. Hattori, S. Li, D. Satow and H.-U. Yee, Longitudinal Conductivity in Strong Magnetic Field in Perturbative QCD: Complete Leading Order, Phys. Rev. D 95 (2017) 076008 [arXiv: 1610.06839] [INSPIRE].

[38] K. Hattori, X.-G. Huang, D.H. Rischke and D. Satow, Bulk Viscosity of Quark-Gluon Plasma in Strong Magnetic Fields, Phys. Rev. D 96 (2017) 094009 [arXiv:1708. 00515] [InSPIRE].

[39] H.-T. Ding, O. Kaczmarek and F. Meyer, Thermal dilepton rates and electrical conductivity of the QGP from the lattice, Phys. Rev. D 94 (2016) 034504 [arXiv:1604.06712] [InSPIRE].

[40] S. Gupta, The Electrical conductivity and soft photon emissivity of the QCD plasma, Phys. Lett. B 597 (2004) 57 [hep-lat/0301006] [INSPIRE].

[41] G. Aarts, C. Allton, J. Foley, S. Hands and S. Kim, Spectral functions at small energies and the electrical conductivity in hot, quenched lattice QCD, Phys. Rev. Lett. 99 (2007) 022002 [hep-lat/0703008] [INSPIRE].

[42] H.T. Ding, A. Francis, O. Kaczmarek, F. Karsch, E. Laermann and W. Soeldner, Thermal dilepton rate and electrical conductivity: An analysis of vector current correlation functions in quenched lattice QCD, Phys. Rev. D 83 (2011) 034504 [arXiv:1012.4963] [INSPIRE].

[43] V.P. Gusynin, V.A. Miransky and I.A. Shovkovy, Dimensional reduction and dynamical chiral symmetry breaking by a magnetic field in $(3+1)$-dimensions, Phys. Lett. B 349 (1995) 477 [hep-ph/9412257] [INSPIRE]. 
[44] K. Fukushima and Y. Hidaka, Magnetic Catalysis Versus Magnetic Inhibition, Phys. Rev. Lett. 110 (2013) 031601 [arXiv: 1209.1319] [INSPIRE].

[45] K. Hattori and K. Itakura, Vacuum birefringence in strong magnetic fields: (I) Photon polarization tensor with all the Landau levels, Annals Phys. 330 (2013) 23 [arXiv: 1209.2663] [INSPIRE].

[46] K. Hattori and K. Itakura, Vacuum birefringence in strong magnetic fields: (II) Complex refractive index from the lowest Landau level, Annals Phys. 334 (2013) 58 [arXiv: 1212.1897] [INSPIRE].

[47] Y. Hidaka and T. Kunihiro, Renormalized Linear Kinetic Theory as Derived from Quantum Field Theory: A Novel diagrammatic method for computing transport coefficients, Phys. Rev. D 83 (2011) 076004 [arXiv: 1009.5154] [INSPIRE].

[48] N. Weickgenannt, X.-L. Sheng, E. Speranza, Q. Wang and D.H. Rischke, Kinetic theory for massive spin-1/2 particles from the Wigner-function formalism, Phys. Rev. D 100 (2019) 056018 [arXiv: 1902.06513] [INSPIRE].

[49] J.-H. Gao and Z.-T. Liang, Relativistic Quantum Kinetic Theory for Massive Fermions and Spin Effects, Phys. Rev. D 100 (2019) 056021 [arXiv: 1902.06510] [INSPIRE].

[50] K. Hattori, Y. Hidaka and D.-L. Yang, Axial Kinetic Theory and Spin Transport for Fermions with Arbitrary Mass, Phys. Rev. D 100 (2019) 096011 [arXiv:1903.01653] [INSPIRE].

[51] Z. Wang, X. Guo, S. Shi and P. Zhuang, Mass Correction to Chiral Kinetic Equations, Phys. Rev. D 100 (2019) 014015 [arXiv: 1903.03461] [INSPIRE].

[52] Y. Minami and Y. Hidaka, Relativistic hydrodynamics from the projection operator method, Phys. Rev. E 87 (2013) 023007 [arXiv:1210.1313] [inSPIRE].

[53] H.B. Nielsen and M. Ninomiya, Adler-Bell-Jackiw anomaly and Weyl fermions in crystal, Phys. Lett. 130B (1983) 389 [INSPIRE].

[54] P. Aurenche and T. Becherrawy, A Comparison of the real time and the imaginary time formalisms of finite temperature field theory for 2, 3 and 4 point Green's functions, Nucl. Phys. B 379 (1992) 259 [INSPIRE].

[55] M.A. van Eijck, R. Kobes and C.G. van Weert, Transformations of real time finite temperature Feynman rules, Phys. Rev. D 50 (1994) 4097 [hep-ph/9406214] [INSPIRE]. 